Cómo citar este trabajo: Cerano Paredes, J., Esquivel Arriaga, G., Sánchez Cohen, I., \& Estrada Arrellano, J. R. (2020). Analysis of meteorological droughts in the Yaqui River Basin, Mexico and its relationship with ENSO. Boletín de la Asociación de Geógrafos Españoles, (86). https://doi.org/10.21138/bage.2937

\title{
Análisis temporal de sequías meteorológicas en la cuenca del Río Yaqui (México) y su relación con ENSO
}

Analysis of meteorological droughts in the Yaqui River Basin, Mexico and its relationship with ENSO

\author{
Julián Cerano Paredes \\ cerano.julian@inifap.gob.mx \\ INIFAP CENID-RASPA (México) \\ Gerardo Esquivel Arriaga \\ esquivel_ag3@hotmail.com \\ INIFAP CENID-RASPA (México) \\ Ignacio Sánchez Cohen \\ sanchez.ignacio@inifap.gob.mx \\ INIFAP CENID-RASPA (México) \\ José Raymundo Estrada Arrellano \\ j.estradarellano@gmail.com \\ UJED Facultad de Ciencias Biológicas (México)
}

\section{Resumen}

Este estudio analiza el comportamiento temporal de las sequías en la Cuenca del Río Yaqui (CRY), México, usando el Índice de Precipitación Estandarizado (SPI, siglas en inglés) y 
determina la influencia del fenómeno climático El Niño Southern Oscillation (ENSO) en la modulación de su variabilidad. Se utilizaron 28 estaciones climáticas distribuidas en el gradiente de la cuenca y $20 \mathrm{~km}$ adicionales al parteaguas. Se evaluó la calidad de las series mediante pruebas de homogeneidad de varianza, se determinó su variabilidad común mediante un Análisis de Componentes Principales (ACP) y se generó un SPI regional para el periodo 19372016. Los eventos de sequía más severos se registraron en los años de 1980, 1988, 1999, 2006 y 2011. El análisis espectral de coherencia de ondeletas entre el SPI y ENSO indica una relación en fase entre eventos secos y lluviosos durante el período de 1937 a 1959 a frecuencias de uno a seis años, de 1937 a 1950 en frecuencias de 10 a 11 años, de 1972 a 1974 a frecuencias de uno a dos años y de 1997 a 2005 a frecuencias de 11 a 15 años. ENSO influye significativamente en la variabilidad del SPI en la cuenca.

Palabras clave: precipitación; índice; sequía; vulnerabilidad climática; hidrología.

\begin{abstract}
This study analyzes the temporal behavior of the droughts in the Yaqui River Basin, in Mexico using the Standardized Precipitation Index (SPI) and determines the influence of the El Niño Southern Oscillation (ENSO) climatic phenomenon in the modulation of the SPI variability. 28 climatic stations distributed in the basin gradient and in an additional area to divide of $20 \mathrm{~km}$ were used. The quality of the series was evaluated by tests of homogeneity of variances, the common variability between stations was determined through a Principal Component Analysis and a regional SPI was generated for the period 1937-2016. The most severe drought events were recorded in the years 1980, 1988, 1999, 2006 and 2011. The spectral coherence of waves analysis between the SPI and the ENSO indicates a phase relationship for dry and rainy events during the period from 1937 to 1959 at frequencies of one to six years, from 1937 to 1950 at frequencies of 10 to 11 years, from 1972 to 1974 at frequencies of one to two years and from 1997 to 2005 at frequencies of 11 to 15 years. ENSO significantly influences the variability of SPI in the basin.
\end{abstract}

Key words: rainfall; index; drought; climatic vulnerability; hydrology.

\title{
1 Introducción y objetivos
}

La sequía es un fenómeno natural que se origina por deficiencias en la precipitación durante un tiempo prolongado y es una anomalía temporal que puede suceder en casi todas las zonas 
climáticas, incluso en aquellas con elevadas precipitaciones (Morales, Olcina \& Rico, 2000). La sequía es un evento extremo y un fenómeno progresivo que, de acuerdo con su intensidad y frecuencia, afecta el medio ambiente e incluso puede causar pérdidas de vidas humanas y daños en la economía, lo cual implica dedicar cuantiosos recursos a su recuperación (Zucarelli, 2013; Mesbahzadeh, Mirakbar, Saravi, Sardoo \& Miglietta, 2020). En la actualidad, ha aumentado la preocupación en todo el mundo de que las sequías puedan aumentar en frecuencia, severidad y duración debido a las condiciones climáticas cambiantes y al incremento de eventos climáticos extremos documentados (Peterson, Hoerling, Stott \& Herring, 2013; Sivakumar et al., 2014).

Hasta el año de 1985 se habían identificado más de 150 definiciones de sequía publicadas en la literatura (Wilhite \& Glantz, 1985) y actualmente existen muchas más. Sin embargo, en los últimos años ha existido un consenso generalizado entre la comunidad científica, quienes aceptan que las definiciones de sequía pueden agruparse en cuatro tipos básicos: meteorológica, agrícola, hidrológica y socioeconómica. La sequía meteorológica (basada en datos climáticos), es una expresión de la desviación de la precipitación respecto a la media durante un período de tiempo determinado. Ante la dificultad de establecer una duración y magnitud del déficit pluviométrico válidas para diferentes áreas geográficas, algunas definiciones de sequía meteorológica optan por no especificar umbrales fijos, mientras que en otros casos las definiciones presentan información específica para cada región en particular, que varían en función de las características del clima regional (Marcos, 2001).

La sequía agrícola se define habitualmente en términos de la disponibilidad de agua en los suelos para el sostenimiento de los cultivos, es una desviación de los regímenes de precipitación normales durante cierto período de tiempo. Para algunos especialistas, el déficit de humedad en el suelo que está ligado a los efectos sobre la producción vegetal -agricultura y pastizales en ganadería, es frecuentemente denominado como sequía edáfica- (Bootsma, Boisvert \& Baier, 1996; Barakat \& Handoufe, 1998). La agricultura es, por tanto, el primer sector económico afectado por la sequía, debido a que el suministro de humedad del suelo a las plantas se agota rápidamente. Los impactos de la sequía en el sector agrícola están vinculados con diversas características de la sequía meteorológica, centrándose en la escasez de precipitaciones, las diferencias entre la evapotranspiración real y potencial, y el déficit de agua del suelo (Ortega, 2012). 
La sequía hidrológica hace referencia a una deficiencia en el caudal o volumen de aguas superficiales o subterráneas (ríos, embalses, acuíferos, lagos, etc.) con respecto a los niveles considerados como normales (Marcos, 2001; Ortega, 2012). Aunque la causa principal de la sequía hidrológica es la sequía meteorológica, lo cierto es que, el concepto de sequía hidrológica es independiente del déficit de precipitación ya que suele definirse como la desviación de las pautas de flujo de agua en la superficie y en el subsuelo, tomando como referencia valores promediados en distintas fechas cronológicas (OMM, 2006). A diferencia de la sequía agrícola, que tiene lugar poco tiempo después de la meteorológica, la sequía hidrológica puede demorarse durante meses desde el inicio de la escasez pluviométrica o, si las Iluvias retornan en poco tiempo, puede ser que no se manifieste (Entekhabi, Rodríguez \& Brass, 1992).

La sequía en el sector socioeconómico ocurre cuando las lluvias son escasas o nulas durante un tiempo lo suficientemente prolongado como para producir sequía agrícola e hidrológica, lo que repercute tanto en daños económicos como personales. La constante presión antropogénica sobre el recurso hídrico hace que sea cada vez mayor la incidencia de la sequía socioeconómica, con pérdidas económicas crecientes, incluso en el caso de una sequía meteorológica leve (Marcos, 2001).

Todos los tipos de sequía son consecuencia de un déficit de precipitación o de una sequía meteorológica, la cual es causada principalmente por la variabilidad natural del clima, que a su vez, puede provocar otros tipos de sequía y de impactos asociados al fenómeno (Ortega, 2012). Por tanto, la sequía meteorológica, sigue siendo la más analizada a escala global (Mirakbari, Ganji \& Fallah, 2010; Modaresi, Ghahraman, Khalili, Ghahremani \& Ahmadi, 2017; Yacoub \& Tayfur, 2017; Zhong, Hua \& Yan, 2020).

Las causas de este fenómeno se agrupan en dos grandes categorías: 1) las de origen natural, representadas por las modificaciones en los patrones de la circulación atmosférica, variaciones en la actividad solar y los fenómenos de interacción entre el océano y la atmósfera, como ENSO, entre otros; y 2) las de origen antropogénico, constituidas por la quema de combustibles fósiles, la degradación ambiental (deforestación, degradación del suelo y desertificación) y la alteración de los sistemas ecológicos naturales (Ortega, 2012).

El fenómeno climático ENSO, es un mecanismo de forzamiento climático que modifica los patrones normales del clima a escala global (Seager et al., 2009) y tiene una gran influencia en la variabilidad de la precipitación en México (Magaña, Vásquez, Pérez y Pérez, 2003). Las dos 
fases de ENSO (El Niño y La Niña) propician un efecto de dipolo en el clima de nuestro país; El Niño está asociado a condiciones de mayor humedad en el norte de México y condiciones de sequía en el centro y sur del país; mientras que La Niña, se asocia a condiciones de sequía para la región norte y mayor humedad para el centro-sur del país (Magaña et al., 2003; Seager et al., 2009; Stahle et al., 2011). Estudios dendroclimáticos han determinado una influencia significativa de ENSO en la variabilidad de la precipitación tanto en el centro como en el norte del país (Stahle et al., 2011; Cerano et al., 2011).

La predicción de condiciones de sequía en el futuro es considerada un paso primordial para la prevención de daños (Mesbahzadeh et al., 2020), por ello, es indispensable analizar la variabilidad interanual de la precipitación, para detectar la presencia de eventos extremos tanto secos como húmedos, así como su recurrencia. Sin embargo, la determinación de las características de la sequía es muy difícil, dado que existe una variedad de índices para su evaluación (Heim, 2002). El SPI (McKee, Doesken \& Kleist, 1993) es el indicador comúnmente usado para analizar la severidad, duración y frecuencia de las sequías meteorológicas (Mesbahzadeh, 2020) y ha permitido detectar, el inicio, fin, intensidad, duración y frecuencia de eventos extremos (sequías e inundaciones) en distintas regiones del Mundo (Heim, 2002; Velasco, 2006; Zucarelli, 2013; Raziei et al., 2014; Paredes, Barbosa \& Guevara, 2015) y de México (Rivera et al., 2007; Nuñez, Muñoz, Reyes, Velasco \& Gadsden, 2007; Escobar, González, Sánchez, Muñoz \& Villa, 2012; Ravelo, Sanz \& Douriet, 2014; Serrano, Vicente, Flores, Tijerina \& Vázquez, 2016; Castillo, Ibañez, Valdés, Arteaga \& Vázquez, 2017; Esquivel et al., 2019).

México, dada su posición geográfica y una compleja topografía, se caracteriza por una gran variabilidad climática (Davidova, 2010). Dichas condiciones (orográficas y climáticas) han creado divisiones naturales del paisaje en una gran diversidad de tamaños y formas, desde miles de kilómetros (cuenca del río Bravo con $222500 \mathrm{~km}^{2}$ ) hasta de un kilómetro cuadrado (cuenca Caleta el Pando, Sonora) (Cotler, 2010). En la parte noroeste del país, se encuentra la Cuenca del Río Yaqui (CRY), la cual es la más grande de esta región, tanto en términos de su extensión (74 $640 \mathrm{~km}^{2}$ ) como por el volumen de sus escurrimientos (2404 millones de metros cúbicos al año) (INEGI, 2000; Lutz \& Curl, 2012; CONAGUA, 2018). Aunque la CRY se caracteriza por su clima semiárido, es una región de gran importancia económica para México, principalmente por su producción agrícola. Presenta un complejo de tres presas construidas en el gradiente de la cuenca que proveen el recurso hídrico para la agricultura en la parte baja, específicamente en los Distritos de Riego (DR) 018 (Colonias Yaquis) y 041 (Rio Yaqui) (2018). 
A pesar de la extensión territorial de la cuenca y de su importancia en la economía para esta región del norte de México, son pocos los estudios que han analizado la variabilidad de la precipitación de la cuenca en términos de sequía. La primera aproximación fue realizada por Nicholas y Battisti (2008) los cuales caracterizaron los eventos secos a una escala temporal de 10 años, utilizando series de precipitación generados a través de modelos de circulación general (Kaplan et al., 1998). Sin embargo, no utilizaron series de precipitación propias de la cuenca, e índices para categorizar la severidad de los eventos de sequía en la zona de estudio. Por otro lado, Martínez y Patiño (2014) analizaron las tendencias de los registros de cuatro estaciones en la parte baja de la cuenca (precipitación y temperatura) encontrando tendencias claras de incrementos de temperatura, no así en el caso de la precipitación, en la que no se observa una tendencia significativa.

Ante tales circunstancias y dada la relevancia de la CRY tanto en aspectos ambientales como económicos para México, y ante una creciente incertidumbre climática en términos de vulnerabilidad en la cuenca, los objetivos planteados en el presente trabajo fueron: 1) desarrollar series de SPI que permitan analizar los eventos de sequía meteorológica en la cuenca del Río Yaqui, México y 2) determinar la influencia de ENSO en la modulación de la variabilidad del SPI.

\section{Datos y metodología}

\section{1 Área de estudio}

La CRY está delimitada por las coordenadas $27^{\circ}$ y $32^{\circ}$ Norte y $107^{\circ}$ y $110^{\circ} 40^{\prime}$ Oeste. De este a oeste, la pendiente predominante varía de fuerte a débil a medida que el río fluye desde su origen hasta el golfo de California. El Río Yaqui, por el cual la cuenca lleva su nombre, constituye uno de los mayores sistemas de ríos del noroeste de México, con una longitud de 397 km y nace en la provincia hidrogeológica de la Sierra Madre Occidental (SMO) (INEGI, 2000). El 82\% de los escurrimientos se producen en la parte alta de la cuenca, en ecosistemas con vegetación de bosque mixto, dichos escurrimientos son conducidos a través de afluentes que se originan tanto en los estados de Sonora como de Chihuahua, y alimentan los distritos de riego (DR) "Río Yaqui" y "Colonia Yaquis", planicies donde se encuentra una población aproximada de 600 mil personas (Vega, Cirett, De la Parra \& Zavala, 2011).

Referente a la producción agrícola, el DR Rio Río Yaqui, presentó una superficie cosechada de 272593 hectáreas (ha) con un valor de la producción de 9958903.12 miles de pesos, mientras 
que el DR Colonias Yaquis fue de 19978 ha sembradas con un valor de 404284.84 miles de pesos, para el ciclo agrícola 2015-2016, siendo el principal cultivo establecido en la región el trigo grano con 165677 ha cosechadas, seguida de maíz grano (15453 ha) y cártamo (11 113 ha). A nivel nacional, el estado de Sonora es el principal productor de trigo grano contribuyendo con el 48 \% de la producción nacional, mientras que la CRY representa el 32.5 \% de dicha producción, lo que determina la relevancia e importancia económica de la región en la producción agrícola de México (CONAGUA, 2017). El área geográfica total o parcial de la CRY está conformada por 33 municipios de Sonora (72\%) y 13 municipios de Chihuahua (18\%) con una población mayor a 1.2 millones (Lutz \& Curl, 2012) (Figura 1).

La CRY ofrece diferentes servicios ambientales como: suministro de agua dulce, la regulación del caudal de los ríos, el mantenimiento de los regímenes hidrológicos naturales, desarrollo de la diversidad biológica, entre otros (Cotler, 2010; Lutz \& Curl, 2012). En términos de biodiversidad, se localizan tres áreas naturales protegidas federales: a) Área de Protección de Flora y Fauna (APFYF) Tutuaca, la cual presenta bosques de pino, pino-encino, encino, pastizales, selva baja y vegetación de galería, esta última se desarrolla linealmente en los márgenes de los ríos y cuya conservación permite mantener la producción de organismos acuáticos, las áreas de aguas subterráneas y la recarga de acuíferos (DOF, 2001), b) Campo Verde, la vegetación dominante se compone de bosque de coníferas, pino-encino y encino, así como especies endémicas de anfibios, mamíferos y reptiles, y otras especies de fauna en peligro de extinción (DOF, 2003; GEC, 2019), y c) Bavispe, la cual se compone de bosque de galería, bosque de pino-encino, bosque de encino, chaparral, matorral subtropical, sarcocrasicaule, desértico micrófilo y espinoso; así como especies de reptiles, aves y mamíferos (DOF, 2017). 
Figura 1. Ubicación geográfica de la Cuenca del Río Yaqui en la región noroeste de México

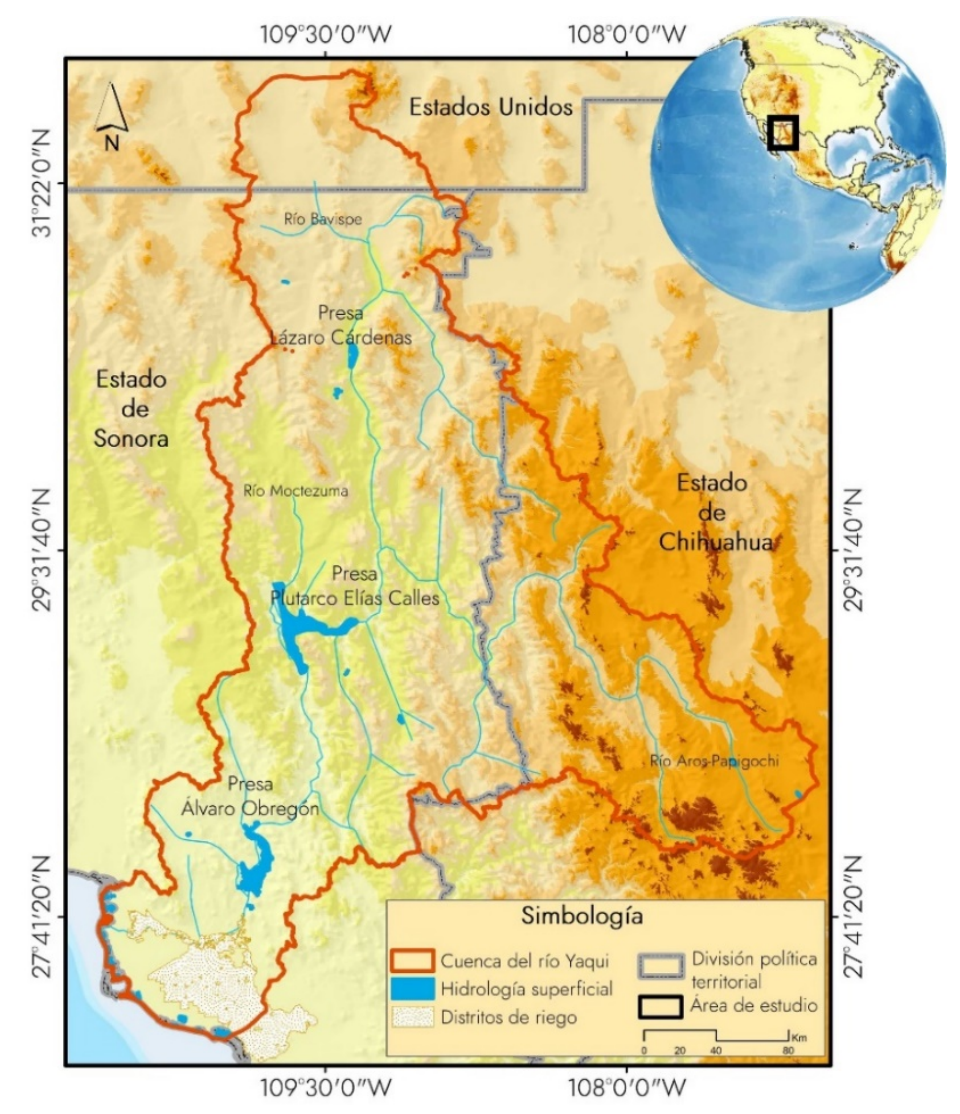

Fuente: elaboración propia con datos de INEGI (2000)

\subsection{Información climatológica}

Las series de datos analizadas se extrajeron del Servicio Meteorológico Nacional (SMN) mediante una aplicación desarrollada para Google Earth, la cual despliega las estaciones meteorológicas de México en dos categorías: operando y suspendidas (SMN, 2019). En la cuenca de estudio y en un área adicional de influencia de $20 \mathrm{~km}$ con relación al parteaguas, se identificó un total de 186 estaciones, de las cuales 113 están suspendidas y 73 continúan en funcionamiento; en este estudio solo se consideraron las estaciones activas. Se analizó la variable precipitación y se eligieron las series que cumplieran con dos criterios básicos establecidos: 1) que las estaciones contaran como mínimo con 30 años de información y 2) que no presentaran más del $10 \%$ de información faltante en su registro histórico. De las 73 estaciones analizadas, 28 cumplieron con los dos criterios establecidos, 21 estaciones dentro de la cuenca y siete en el área de influencia, las cuales presentan diferente periodo histórico de información (Figura 2, Tabla 1). 
Figura 2. Distribución espacial de las estaciones climáticas seleccionadas en la Cuenca del Río Yaqui. La línea punteada representa el área de influencia adicional $(20 \mathrm{~km})$

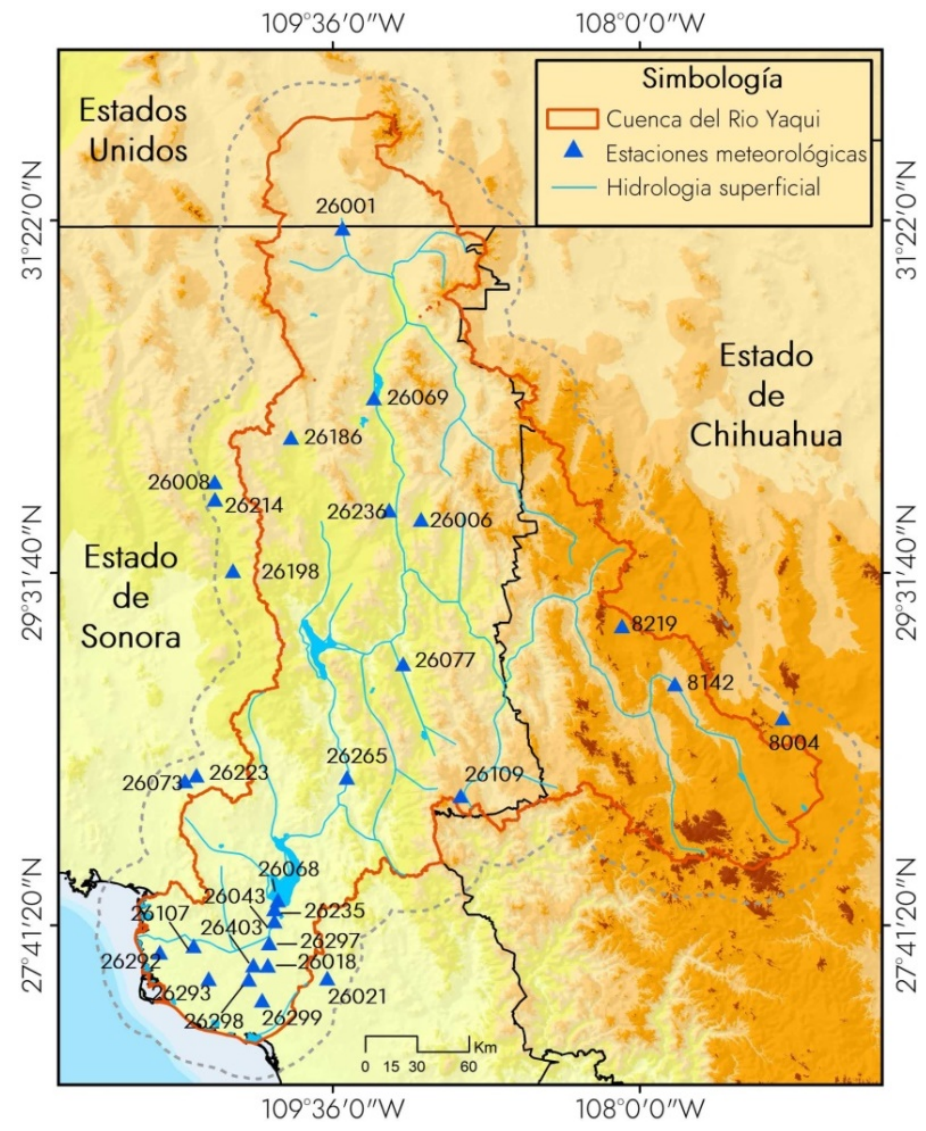

Fuente: elaboración propia con datos de INEGI (2000) y SMN (2019)

Tabla 1. Estaciones meteorológicas que cumplieron los criterios de selección

\begin{tabular}{|r|l|c|c|c|c|c|}
\hline $\begin{array}{c}\text { Clave } \\
\text { SMN }\end{array}$ & \multicolumn{1}{|c|}{$\begin{array}{c}\text { Nombre } \\
\text { de la estación }\end{array}$} & Latitud & Longitud & $\begin{array}{c}\text { Altitud } \\
(\mathrm{m})\end{array}$ & $\begin{array}{c}\text { Precipitación } \\
\text { Media Anual }\end{array}$ & $\begin{array}{c}\text { Amplitud del } \\
\text { registro }\end{array}$ \\
\hline \multicolumn{7}{|c|}{ Estaciones dentro de la cuenca } \\
\hline 8142 & Temosachic & -107.85 & 28.95 & 1990 & 445.40 & $1981-2012$ \\
\hline 8219 & Peñitas & -108.03 & 29.21 & 2212 & 357.70 & $1972-2012$ \\
\hline 26001 & Agua Prieta & -109.56 & 31.33 & 1189 & 355.20 & $1961-2015$ \\
\hline 26006 & Bacadehuachi & -109.28 & 29.76 & 706 & 483.10 & $1963-2015$ \\
\hline 26018 & Cd. Obregón & -109.93 & 27.48 & 100 & 376.90 & $1961-2016$ \\
\hline 26043 & Hornos & -109.90 & 27.71 & 85 & 408.80 & $1960-2016$ \\
\hline 26068 & Presa Alvaro Obregón & -109.90 & 27.81 & 70 & 414.20 & $1949-2016$ \\
\hline 26069 & Presa La Angostura & -109.38 & 30.45 & 965 & 402.20 & $1937-2010$ \\
\hline 26077 & Sahuaripa & -109.23 & 29.05 & 460 & 567.10 & $1942-2016$ \\
\hline 26107 & Vicam & -110.28 & 27.65 & 46 & 337.10 & $1962-2016$ \\
\hline 26109 & Yecora & -108.95 & 28.36 & 1500 & 10360 & $1944-2015$ \\
\hline 26186 & El Tapiro & -109.81 & 30.23 & 890 & 511.60 & $1977-2016$ \\
\hline 26235 & Colonias Yaquis & -109.90 & 27.76 & 47 & 418.20 & $1980-2016$ \\
\hline 26236 & Granados & -109.30 & 29.85 & 533 & 460.50 & $1980-2016$ \\
\hline 26265 & Onavas & -109.53 & 28.46 & 175 & 608.70 & $1982-2016$ \\
\hline
\end{tabular}


Tabla 1. Continuación

\begin{tabular}{|l|l|c|c|c|c|c|}
\hline $\begin{array}{c}\text { Clave } \\
\text { SMN }\end{array}$ & \multicolumn{1}{c|}{$\begin{array}{c}\text { Nombre } \\
\text { de la estación }\end{array}$} & \multicolumn{1}{c|}{ Latitud } & Longitud & $\begin{array}{c}\text { Altitud } \\
(\mathrm{m})\end{array}$ & $\begin{array}{c}\text { Precipitación } \\
\text { Media Anual }\end{array}$ & $\begin{array}{c}\text { Amplitud } \\
\text { del registro }\end{array}$ \\
\hline \multicolumn{7}{|c|}{ Estaciones dentro de la cuenca } \\
\hline 26292 & Guaymas 4-P-6 & -110.24 & 27.42 & 35 & 266.40 & $1967-2007$ \\
\hline 26293 & San lgnacio 4-P-12 & -110.18 & 27.30 & 25 & 290.00 & $1987-2016$ \\
\hline 26297 & Porfirio Díaz & -109.93 & 27.59 & 40 & 375.50 & $1969-2016$ \\
\hline 26298 & Calle seiscientos & -110.03 & 27.40 & 20 & 314.90 & $1968-2016$ \\
\hline 26299 & Calle mil doscientos & -109.96 & 27.30 & 24 & 333.60 & $1980-2016$ \\
\hline 26403 & Calle doscientos & -110.01 & 27.48 & 29 & 284.60 & $1968-2016$ \\
\hline \multicolumn{7}{|c|}{ Area de influencia de la cuenca } \\
\hline 8004 & Bachiniva & -107.25 & 28.80 & 2020 & 409.00 & $1942-2012$ \\
\hline 26008 & Banamichi & -110.21 & 30.01 & 640 & 452.80 & $1961-2016$ \\
\hline 26021 & Cocoraque & -109.61 & 27.40 & 100 & 456.30 & $1969-2015$ \\
\hline 26073 & Punta de agua & -110.43 & 28.41 & 214 & 423.90 & $1957-2015$ \\
\hline 26198 & Mazocahui & -110.11 & 29.53 & 492 & 555.10 & $1982-2016$ \\
\hline 26214 & Huepac & -110.20 & 29.90 & 660 & 514.00 & $1979-2016$ \\
\hline 26223 & Punta de agua & -110.30 & 28.46 & 250 & 452.70 & $1980-2015$ \\
\hline
\end{tabular}

Fuente: Elaboración propia con datos del SMN (2019)

\subsection{Control de calidad de las series de precipitación}

Para validar la consistencia y homogeneidad de las series de datos, se realizó un análisis gráfico y la aplicación de tres pruebas estadísticas de homogeneidad: 1) prueba de homogeneidad normal estándar (SNHT, por sus siglas en inglés) (Alexandersson \& Moeberg, 1997), 2) Pettitt (Pettitt, 1979) y 3) Buishand (Buishand, 1982), las cuales se describen brevemente a continuación, junto con sus estadísticos de prueba.

1) Prueba de Homogeneidad normal estándar (SNHT), desarrollada por Alexandersson (Alexandersson, 1986) y que modificó con Moberg (Alexandersson \& Moeberg, 1997); un valor $T(d)$ compara el promedio de los primeros $d$ años registrados con los últimos $(n-d)$; este valor se obtiene con la expresión:

$$
T_{d}=d \bar{z}_{1}+(n-d) \bar{z}_{2} \text { para } d=1,2,3 \ldots, n
$$

donde:

$$
\bar{z}_{1}=\frac{1}{d} \frac{\sum_{i=1}^{d}\left(y_{i}+\bar{y}\right)}{s} y \frac{1}{n-d} \frac{\sum_{i=d+1}^{n}\left(y_{i}-\bar{y}\right)}{s}
$$

Un valor alto de $T$ en un año $d$ indica una variación "brusca". El estadístico de prueba $T_{0}$ se define como:

$$
T_{o}=\underset{1 \leq d \leq 0}{\operatorname{máx}} T(d)
$$


2) Pettitt, prueba no paramétrica, que se basa en rangos y hace caso omiso de la normalidad de la serie. Se basa en el orden de rangos de los valores yi. El estadístico que se emplea se define como:

$$
X_{d}=2 \sum_{i=1}^{d} r_{i}-d(n+1) \text { para } d=1,2,3, \ldots, n
$$

y un valor en el año m que cumple la condición:

$$
X_{m}=\underset{1 \leq d \leq n}{\operatorname{máx}}\left|X_{d}\right|
$$

es una variación fuerte en la serie (Pettitt, 1979).

3) Buishand, por su origen bayesiano, puede ser empleada en variables con cualquier tipo de distribución y propone identificar un cambio en la media de la serie de datos. El estadístico se define como:

$$
S_{0}^{*}=0 \text { y } S_{d}^{*}=\sum_{i=1}^{d}\left(y_{i}-\bar{y}\right) \text { para } d=1,2,3, \ldots, n
$$

La prueba se define como:

$$
Q=\underset{0 \leq d \leq n}{\operatorname{máx}}\left|\frac{S_{d}^{*}}{S}\right|
$$

Otro estadístico de prueba que puede emplearse es el rango que calcula la diferencia entre el valor mínimo y máximo de las sumas parciales ajustadas. La fórmula es:

$$
R=\frac{\left(\begin{array}{c}
\operatorname{má} x S_{d-}^{*} \min n S_{d}^{*} \\
0 \leq d \leq n \\
0 \leq d \leq n
\end{array}\right)}{S}
$$

Es importante agregar que Buishand propone valores críticos $Q / V n$ y $R / V n$ para las pruebas de homogeneidad (Buishand, 1982; Guajardo, Granados, Sánchez, Díaz \& Barbosa, 2017).

Las hipótesis planteadas para las tres pruebas fue la siguiente: Ho: los datos son homogéneos vs Ha: hay una fecha en la que hay un cambio en los datos $(\alpha=0.05)$. Las pruebas se realizaron mediante el software XLSTAT, donde se obtuvieron los valores p (p-value) correspondientes; los resultados se clasificaron en tres categorías con base en el trabajo de Guajardo et al. (2017). En la categoría uno, se agruparon las series cuyas pruebas de hipótesis nula fueron rechazadas en una de las tres pruebas, por lo que la serie se considera confiable; en la categoría dos, se encuentran las series que presentaron dos hipótesis nulas, la información se clasificó como medianamente confiable y en la categoría tres, se agrupan las series en las que se rechazaron tres hipótesis nulas, por lo cual la información fue considerada como no confiable.

El uso de pruebas estadísticas para el control de calidad a las series de datos, es de vital 
importancia para asistir en la detección de que las variaciones y tendencias en una serie de tiempo analizada, responde exclusivamente al forzamiento de la variabilidad y climática y que, por lo tanto, no están asociadas a otros factores artificiales y espurios, como: los inducidos por cambios en el tiempo en la instrumentación, en las practicas observacionales o en el entorno de los puntos de observación; este conjunto de factores artificiales pueden introducir rupturas puntuales o graduales en la homogeneidad de cualquier serie de tiempo que pueden ser de magnitud similar e incluso mayor que la verdadera señal climática (Brunet, 2010).

\section{4 Índice de precipitación estandarizado}

Para el cálculo del SPI se consideró la metodología descrita por McKee et al. (1993) y se utilizó el software SPI Generator de distribución gratuita (NDMC, 2019). El cálculo del SPI comprende primeramente el ajuste a la función de densidad de probabilidad ( $\mathrm{fdp}$ ) gamma del registro de precipitación para cada estación meteorológica; posteriormente, los valores ajustados se transforman a la fdp normal con media cero y varianza uno (normal estándar), que representa el valor del SPI. Esta estandarización de la variable aleatoria se basa en el principio de equiprobabilidad, el cual establece que el valor de la función de distribución de probabilidad para un valor dado de la variable aleatoria es el mismo para la variable original que para la transformada (Velasco, Aparicio, Valdés, Velázquez y Kim, 2004).

La función de distribución de probabilidad empírica $F(x)$ se encuentra mediante la fórmula de Weibull (Ponce, 1989) ordenando los valores de precipitación por magnitud en forma ascendente de tal forma que para el k-ésimo valor de una muestra de tamaño n (registro):

$$
F(x)=\frac{k}{n+1}
$$

Para periodos de tiempo mayores a un mes, el procedimiento básico inicial para estimar el SPI es tomar sumas móviles, por ejemplo, para escalas de tres meses, la suma de los valores de Iluvia de los primeros tres meses de la serie forman el primer valor de la nueva serie a ajustar a la fdp gamma; la suma de los valores de lluvia del mes dos al cuatro de la serie mensual formara el segundo valor de la serie nueva, y así sucesivamente. Por tanto, si n es el número total de meses de la serie histórica, y $r$ la escala temporal del SPI ( $r=1,2,3,06012$, etcétera), entonces la nueva serie tendrá $n_{r}=n-r+1$ valores. Con lo anterior, al disponer de una serie de tiempo de valores mensuales de precipitación lo suficientemente larga (idealmente de treinta años o mayor, de tal forma que sea representativa de las condiciones de largo plazo) y confiable, es posible calcular el SPI en cualquier mes del registro para los $i$ meses previos, dependiendo de la escala temporal de interés (Velasco et al., 2004; Castillo et al., 2017). 
En este estudio, se calculó el SPI para las 28 series de precipitación de la cuenca a una escala temporal de 12 meses, considerando el periodo histórico de manera independiente para cada serie. Se eligió esta temporalidad dado que, en la escala de tres meses, los valores del SPI presentan alta sensibilidad a los cambios de la precipitación acumulada durante el periodo trimestral, por lo cual, cambian de positivo a negativo en cortos periodos de tiempo. En series de tiempo más extensas (seis, 12 o 24 meses) cada nuevo registro de precipitación tiene menor incidencia en el total acumulado del periodo correspondiente, por lo tanto, los valores del SPI se estabilizan y definen con mayor claridad el déficit de la precipitación (Nuñez et al., 2007).

Asimismo, a escala temporal de 12 meses, tiene la ventaja de poder evaluar el comportamiento anual de la precipitación y no sólo de enero a diciembre, sino para cualquier mes, por ejemplo de octubre a septiembre. Esta escala temporal es útil para evaluar la sequía de mediano plazo y tomarla como referencia al dimensionar los impactos de la falta de agua en los diferentes sectores económicos y sociales, por ejemplo, los daños en el sector ganadero usualmente se reportan y evalúan en términos anuales, así como los de la agricultura de riego, cuando la fuente de suministro es un embalse (Velasco et al., 2004). La categorización del SPI se realizó de acuerdo a la clasificación de la OMM (2012) (Tabla 2).

\section{Tabla 2. Clasificación de los valores del SPI}

\begin{tabular}{|c|c|}
\hline Valor del SPI & Categoría \\
\hline 2.0 o más & Extremadamente húmedo \\
\hline 1.50 a 1.99 & Muy húmedo \\
\hline 1.0 a 1.49 & Moderadamente húmedo \\
\hline-0.99 a 0.99 & Moderadamente seco \\
\hline-1.0 a -1.49 & Severamente seco \\
\hline-1.5 a -1.99 & Extremadamente seco \\
\hline-2.0 o menos & \\
\hline
\end{tabular}

Fuente: OMM (2012)

\subsection{Variabilidad de las series del SPI}

Para determinar la co-variabilidad de los patrones húmedos y secos en la cuenca, se corrió un análisis de correlación de Pearson $(p<0.01)$ y un ACP para las 28 series de SPI; se consideró el periodo común entre estaciones que comprende de 1987 a 2006 (20 años). El ACP es un método estadístico lineal bien establecido, frecuentemente utilizado para analizar datos hidroclimatológicos como series de precipitación (Esquivel et al., 2019) y caudal (Canchala et al., 2020). Su objetivo principal es reducir el tamaño de la serie temporal hacia algunos componentes principales ortogonales (CPs) que explican la mayoría de la variabilidad de las 
variables originales con mínima pérdida de información (Helena et al., 2000; Jolliffe y Cadima, 2016). En el presente estudio, el método de ACP se utilizó para representar y caracterizar los patrones dominantes en la variabilidad del SPI entre estaciones en la CRY, lo cual permitió integrar un SPI regional.

\subsection{Influencia del ENSO en la variabilidad del SPI}

Para determinar la influencia del fenómeno atmosférico circulatorio ENSO en la variabilidad del SPI en la CRY, se relacionó el SPI regional (1937-2016) con el índice Niño 3.4 reconstruido a partir de 2222 cronologías de anillos de árboles a nivel mundial (Li et al., 2013). Se empleó este índice por fundamentarse en una base de datos geográficamente muy amplia, que comprende series de los trópicos y latitudes medias en ambos hemisferios; así mismo, la inclusión de registros tropicales permite alcanzar mayor precisión. Para tal efecto se utilizaron gráficos de resolución anual de espectros de ondeleta y coherencia de ondeletas empleando el software MałLab 6.5 y el código desarrollado por Grinsted et al. (2004). ${ }^{1}$

El análisis de ondeletas se ha utilizado ampliamente en diferentes trabajos de investigación en todo el mundo para analizar el tiempo de ENSO (Torrence \& Compo, 1998). Después de obtener el SPI regional, este fue descompuesto sobre un espacio de tiempo y frecuencia por el análisis de ondeletas de Morlet (Torrence \& Compo, 1998; Andreoli \& Kayano, 2004). La ondeleta de Morlet es un exponencial complejo que es modulado por una ventana gaussiana:

$$
\psi(t)=e^{i \omega_{0} \eta} e-\frac{\eta^{2}}{2}
$$

dónde $\eta=t / s$, $t$ es el tiempo, s es la escala de ondeleta acorde al tiempo ( $s=2 / \partial t)$ y $\omega_{0}$ es una frecuencia no dimensional (Torrence \& Compo, 1988). El espectro potencial de ondeletas global para una escala $s$ dada es el tiempo medio de todos los espectros potenciales de ondeleta y se cuantifica como:

$$
\bar{W}^{2}(s)=\frac{1}{N} \sum_{n=0}^{N-1}\left|W_{n}(s)\right|^{2}
$$

El análisis de coherencia de ondeletas y diferencia de fases fue realizado para comparar el SPI regional y el índice climático ENSO (Li et al., 2013). Dadas dos series de tiempo, $X(t)$ e $Y(t)$, con sus respectivas transformadas de ondeleta, $W^{X}(t, s)$ y $W^{Y}(t, s)$, el espectro de ondeleta

1 El código está disponible en https://noc.ac.uk/business/marine-data-products/cross-wavelet-wavelet-coherencetoolbox-matlab 
cruzada se define como $W^{X Y}(t, s)=W^{X}(t, s) W^{Y}(t, s)$, donde $\left({ }^{*}\right)$ indica el complejo conjugado. La coherencia de la ondeleta cuadrada se define como el cuadrado del suavizado del módulo del espectro de ondeleta cruzado normalizado por el espectro de ondeleta suavizado:

$$
R_{n}^{2}(s)=\frac{\left|\left\langle s^{-1} W^{X Y}(t, s)\right\rangle\right|^{2}}{\left\langle s^{-1}\left|W^{X}(t, s)\right|^{2}\right\rangle\left\langle s^{-1}\left|W^{Y}(t, s)\right|^{2}\right\rangle}
$$

donde \langle\rangle es un suavizado en tiempo y escala. El factor $s^{-1}$ es usado para convertir el cuadrado de la coherencia de ondeleta dentro de la densidad de energía. La coherencia de ondeletas precisamente representa la covarianza (normalizada) entre ambas series de tiempo debido a que la transformada de ondeletas mantiene la varianza (Torrence \& Compo, 1988). La diferencia de fase en la coherencia de ondeletas se indica de la siguiente manera:

$$
\Phi_{n}(s)=\tan ^{-1}\left(\frac{\mathfrak{T} m\left\{\left\langle s^{-1} W^{X Y}(t, s)\right\rangle\right\}}{\mathfrak{R e}\left\{\left\langle s^{-1} W^{X Y}(t, s)\right\rangle\right\}}\right)
$$

donde $(\mathfrak{I} m)$ y $(\mathfrak{R} e)$ son las partes imaginarias y reales de $W^{X Y}(t, s)$, respectivamente. El nivel de significancia estadística de la coherencia de ondeletas debe ser determinada utilizando los métodos de Monte Carlo (Grinsted, Moore \& Jevrejeva, 2004).

\section{Resultados y discusión}

\subsection{Variabilidad de la precipitación en la cuenca}

Para la CRY la precipitación media anual (PMA) es de 443 mm (1973-2016) con una desviación estándar de $146 \mathrm{~mm}$. El ciclo anual de la precipitación indica que la estación de verano (junio a septiembre) representa el $71 \%$ de la lluvia total en la cuenca, mientras que el invierno (diciembre-marzo) constituye el $17 \%$ y el otro $12 \%$ se distribuye durante el resto del año (Figura 3). Esta distribución corrobora lo indicado por García (2003) la cual analizó la distribución de la precipitación para la república mexicana, identificando los principales regímenes pluviométricos, de los cuales la región de estudio presenta el de lluvias de verano.

Aunque la CRY es una región muy extensa y con cambios importantes en elevación y orografía, generalmente se caracteriza por su clima semiárido (Martinez, Patiño \& Tamayo, 2014) salvo por la región sur-sureste, que representa las partes más altas de la cuenca y donde se registran los valores máximos de precipitación media anual (PMA). En la porción este de la cuenca se localizan las cuatro estaciones climatológicas con el mayor gradiente altitudinal (1600-2200 
msnm), Peñitas (8219), Bachiniva (8004) y Temósachic (8142) correspondientes al estado de Chihuahua y Yecora (26109) del estado de Sonora (Figura 4a), ésta última con el valor máximo de PMA de 1036 mm en toda la cuenca.

Figura 3. Ciclo anual de la precipitación en la cuenca del río Yaqui de 1937-2016

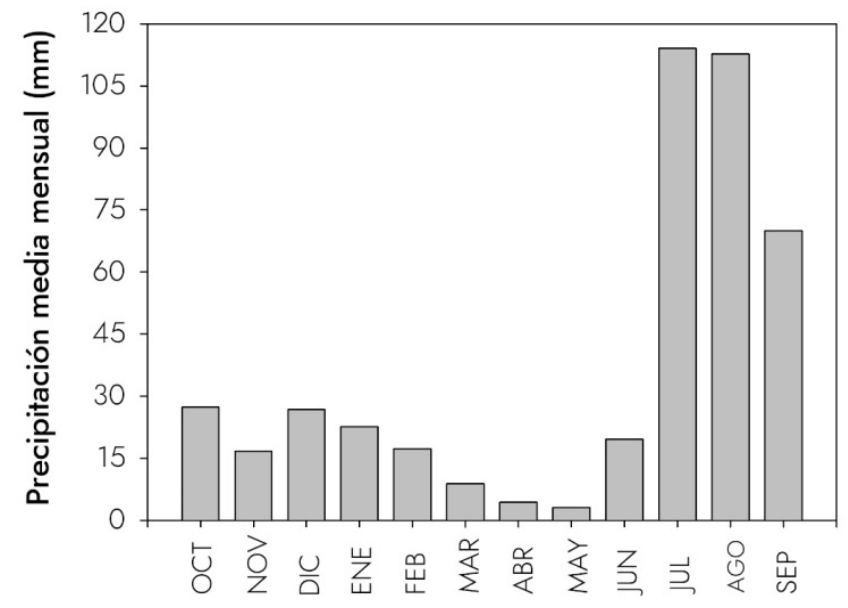

Fuente: elaboración propia a partir del SMN (2019)

Figura 4. Distribución altitudinal de las estaciones climatológicas de la CRY (a) e isoyetas interanuales con la ubicación espacial de las estaciones analizadas en este estudio (b)
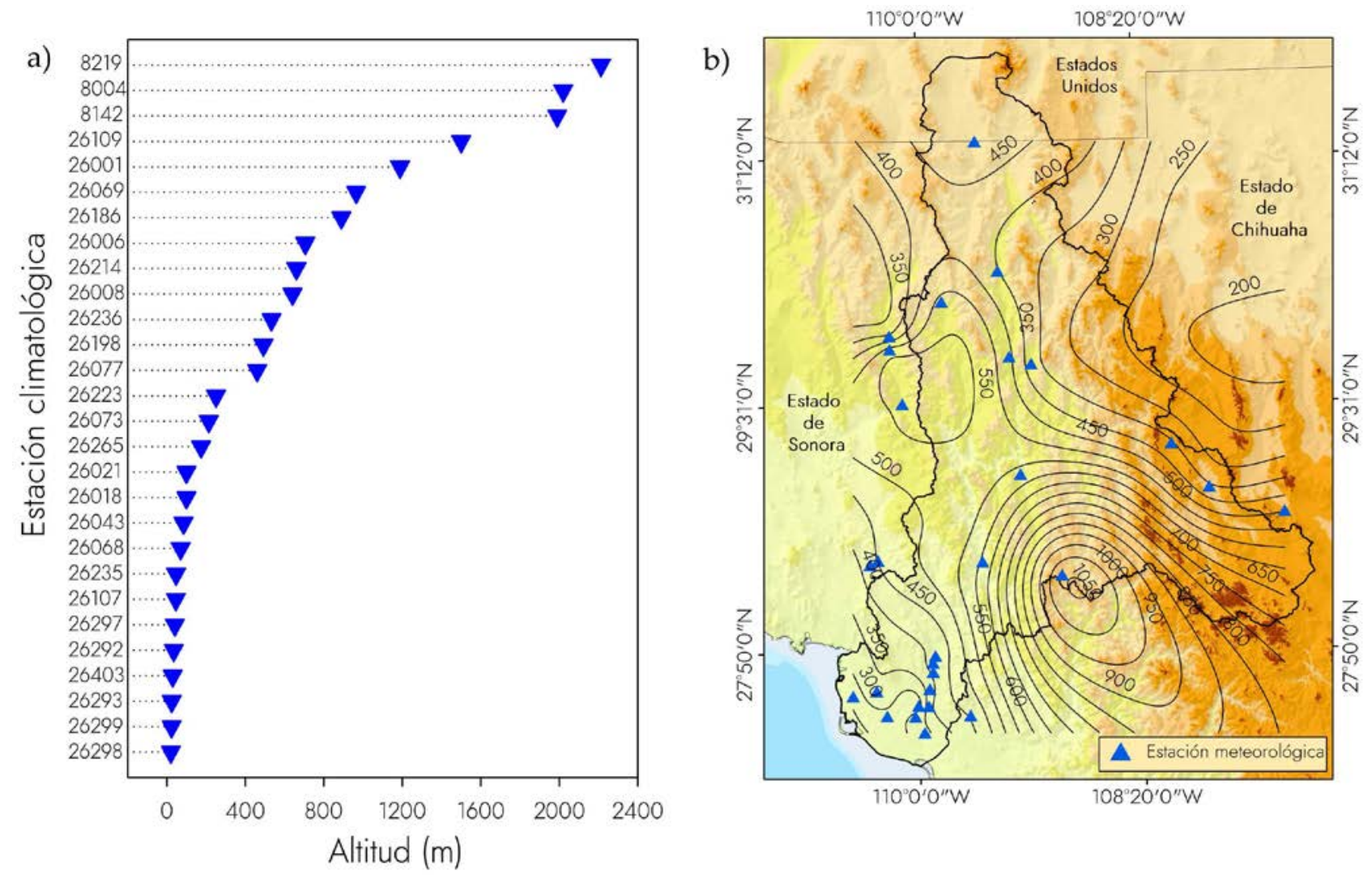

Fuente: elaboración propia a partir del SMN (2019) 
La segunda y tercera serie con los mayores registros de PMA corresponden a la estación Onavas (26265) y Sahuaripa (26077) con 608 y 567 mm, respectivamente; sin embargo, existe una marcada diferencia en cuanto al régimen de precipitación con respecto a la estación Yecora (26109) que registra 1036 mm (Figura 5). Una primera impresión indica que el valor de precipitación de esta última estación presenta un error; sin embargo, un análisis de los valores de precipitación de estaciones vecinas muestra valores similares en el régimen pluviométrico (SMN, 2019). A pesar de que existe un gran número de estaciones que han dejado de funcionar en los últimos años dentro de la superficie que comprende la CRY (SMN, 2019), para las estaciones donde se han registrado valores atípicos se ha logrado validar su calidad, lo cual ha permitido tomar la decisión de considerar dichos eventos extremos en el análisis o excluirlos para una mayor confiabilidad de la distribución de la lluvia.

La distribución de las máximas precipitaciones anuales denotan una clara concentración en la porción sur-sureste (con valores de entre 900 y 1000 mm), con un patrón de disminución hacia el oeste y suroeste de la cuenca (partes bajas), donde se registran precipitaciones del orden de los 300 mm anuales (Figura 4b). Esta distribución es similar a lo analizado por Descroix, Nouvelot, Estrada y Gutiérrez (2004) para la cuenca alta del Río Nazas en el estado de Durango, dónde se presenta el mismo patrón de distribución de la lluvia, pero en la vertiente este de la SMO.

Figura 5. Diagrama de cajas de la precipitación media anual para cada estación climatológica. La media se indica con una línea horizontal en color negro al centro, las desviaciones estándar con líneas horizontales a los extremos y los círculos negros indican valores extremos

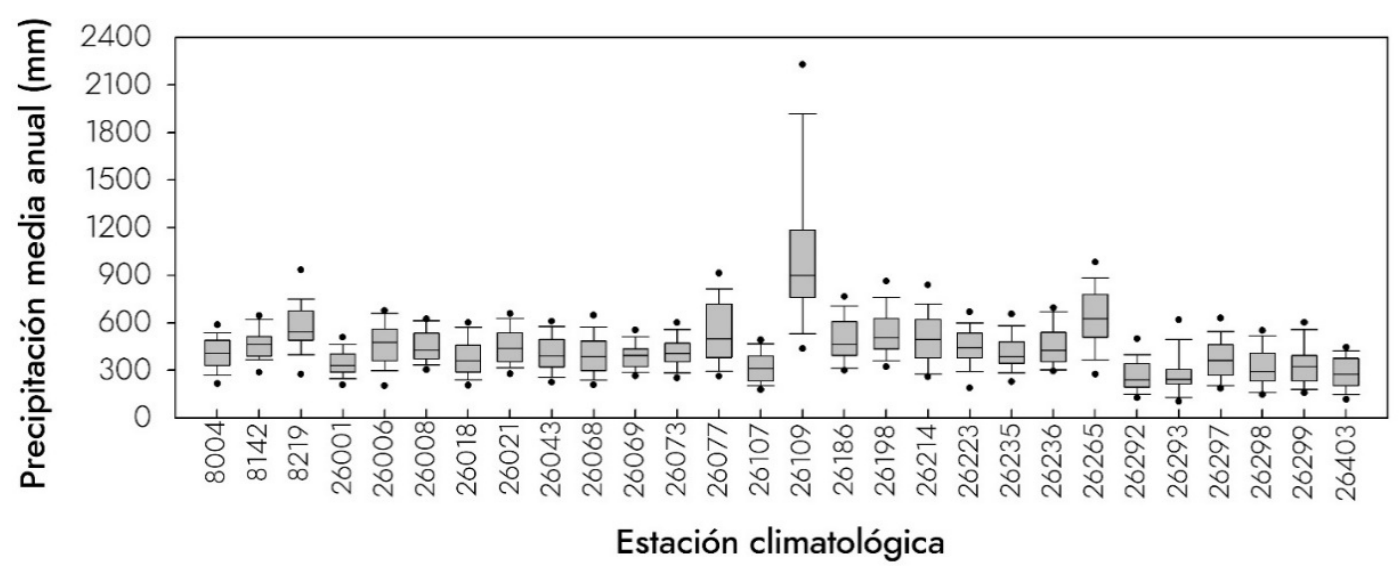

Fuente: elaboración propia a partir de las bases analizadas del SMN (2019) 


\subsection{Pruebas de homogeneidad de varianzas}

El $96 \%$ de las series analizadas presentaron condiciones de homogeneidad con base en las categorías establecidas. Únicamente para dos series se rechazó Ho: la serie de la estación Sahuaripa (26077) bajo la prueba de Pettitt y Buishand, dicha estación se consideró como medianamente confiable y fue descartada para los análisis posteriores, y la estación Yecora (26109) bajo la prueba de Buishand, que acorde a las categorías determinadas fue considerada confiable (Tabla 3). Guajardo et al. (2017) emplearon las mismas tres pruebas de homogeneidad que se utilizaron en este estudio para validar e identificar registros anómalos de precipitación, temperatura máxima y temperatura mínima diaria en el estado de Veracruz, México. De igual manera, Hainie y Sayang (2013) consideraron las las mismas tres pruebas para detectar inhomogeneidad en series de precipitación y número de días húmedos en Malasia peninsular.

Tabla 3. Pruebas de homogeneidad de varianzas realizadas a 28 series de precipitación. Los valores remarcados en color negro son las pruebas en las cuales se rechazó la hipótesis nula

\begin{tabular}{|l|l|c|c|c|}
\hline \multicolumn{1}{|c|}{$\begin{array}{c}\text { Clave } \\
\text { SMN }\end{array}$} & Nombre de la estación & \multicolumn{3}{|c|}{ Pruebas de homogeneidad (valor de P) } \\
\hline \multicolumn{4}{|c|}{ Estaciones dentro de la cuenca } \\
\hline \multicolumn{4}{|c|}{ SNHT } & Buishand's \\
\hline 8142 & Temosachic & 0.131 & 0.874 & 0.496 \\
\hline 8219 & Peñitas & 0.014 & 0.257 & 0.050 \\
\hline 26001 & Agua Prieta & 0.048 & 0.740 & 0.309 \\
\hline 26006 & Bacadehuachi & 0.054 & 0.399 & 0.940 \\
\hline 26018 & Cd. Obregón & 0.641 & 1.000 & 0.990 \\
\hline 26043 & Hornos & 0.322 & 0.937 & 0.591 \\
\hline 26068 & Presa Álvaro Obregón & 0.770 & 0.395 & 0.658 \\
\hline 26069 & Presa La Angostura & 0.214 & 0.659 & 0.234 \\
\hline 26077 & Sahuaripa & $<0.0001$ & 0.001 & $<0.0001$ \\
\hline 260107 & Vicam & 0.737 & 0.682 & 0.360 \\
\hline 26109 & Yecora & 0.151 & 0.018 & 0.0002 \\
\hline 26186 & El Tapiro & 0.463 & 0.921 & 0.564 \\
\hline 26235 & Colonias Yaquis & 0.056 & 0.885 & 0.506 \\
\hline 26236 & Granados & 0.016 & 0.173 & 0.032 \\
\hline 26265 & Onavas & 0.431 & 0.602 & 0.550 \\
\hline 26292 & Guaymas 4-P-6 & 0.294 & 0.990 & 0.936 \\
\hline 26293 & San lgnacio 4-P-12 & 0.095 & 0.264 & 0.225 \\
\hline 26297 & Porfirio Díaz & 0.511 & 0.340 & 0.130 \\
\hline 26298 & Calle seiscientos & 0.495 & 0.925 & 0.990 \\
\hline 26299 & Calle mil doscientos & 0.653 & 0.951 & 0.967 \\
\hline 26403 & Calle doscientos & 0.907 & 0.995 & 0.956 \\
\hline
\end{tabular}


Tabla 3. Continuación

\begin{tabular}{|l|l|c|c|c|}
\hline $\begin{array}{l}\text { Clave } \\
\text { SMN }\end{array}$ & Nombre de la estación & $\begin{array}{c}\text { Pruebas de } \\
\text { homogeneidad } \\
\text { (valor de P) }\end{array}$ & Clave SMN & $\begin{array}{c}\text { Nombre de } \\
\text { la estación }\end{array}$ \\
\hline \multicolumn{4}{|c|}{ Área de influencia de la cuenca } \\
\hline \multicolumn{4}{|c|}{ Pettitt's } \\
\hline 8004 & Bachiniva & 0.275 & 0.986 & 0.771 \\
\hline 26008 & Banamichi & 0.948 & 0.976 & 0.975 \\
\hline 26021 & Cocoraque & 0.489 & 0.932 & 0.997 \\
\hline 26073 & Punta de agua & 0.451 & 0.868 & 0.791 \\
\hline 26198 & Mazocahui & 0.117 & 0.799 & 0.509 \\
\hline 26214 & Huepac & 0.050 & 0.703 & 0.845 \\
\hline 26223 & Punta de agua & 0.992 & 0.879 \\
\hline
\end{tabular}

Fuente: elaboración propia a partir de las pruebas de homogeneidad realizadas a las series de datos obtenidas del SMN (2019)

\section{3 Índice estandarizado de precipitación}

Los resultados indican que 27 series presentan consistencia y homogeneidad en sus datos, por lo tanto, se generaron series de SPI a una escala temporal de 12 meses. Se identificaron los años de eventos moderados, severos y extremos de sequía entre las series de precipitación. Se detectó variabilidad en la longitud de registro de información entre las series analizadas, series de SPI de 76 y 72 años de longitud (Presa la Angostura, 26069; Yecora 26109, respectivamente) y series que apenas alcanzan 30 años de registros (San Ignacio 4-p-12, 26293).

Acorde al índice de severidad de sequía, se identificaron seis años con eventos extremadamente secos en la CRY, el primero de ellos ocurrió en el año de 1980 y se registró en cuatro estaciones $(26018,26107,26297$ y 26403) con valores de -2.2 a -2.5 , mientras que para el resto de las estaciones la severidad de sequía fluctuó de moderada a severa. Estas cuatro estaciones se localizan en la parte baja de la cuenca entre los 29 y 100 msnm, por lo que el impacto de este evento se agudizó más en esta porción de la cuenca (Figura 6). El segundo evento extremo se presentó únicamente en la estación Ciudad Obregón (26018) en el año 1988 (Figura 6).

El tercer y cuarto año extremo de aridez se registró en los años 1989 y 1990, presente únicamente en la estación Yecora (26109) con valores de -2.2 y -2.4, respectivamente. Cabe destacar que, para estos años la frecuencia de sequías moderadas o severas no sincroniza con las demás series de precipitación (Figura 6). Cabe mencionar, que la estación Yecora (26109) se 
ubica a 1500 msnm hacia el sureste de la cuenca entre los límites de Sonora y Chihuahua, con una PMA de 1036 mm, lo cual representa la estación con el mayor régimen pluviométrico de toda la cuenca del rio Yaqui (Figura 5).

Para verificar lo anterior, se revisaron los registros de lluvia de estaciones vecinas durante los mismos años (1989 y 1990) para obtener información que permitiera analizar la PMA y compararla entre ellas; solamente dos estaciones (suspendidas) presentaron información para los años 1989 y 1990, Yecora DGE-26123 (PMA de 993 mm) ubicada a sólo1km de distancia y Ciudad Cubil-26029 (PMA de $623 \mathrm{~mm}$ ) ubicada a 34 km en dirección norte. Para la primera estación, los registros de PMA para los años 1989 y 1990 fue de 633.9 mm y 1261.9 mm, mientras que para la segunda estación fue de 675 y 1130 m; mientras que para la estación Yecora (26109) el régimen fue de 171.5 mm y 97.1 mm, para 1989 y 1990, respectivamente.

Figura 6. Años que presentaron evento de sequía acorde al valor del SPI.

Las columnas remarcadas en color negro indican los años en los cuales hubo una mayor sincronía de eventos entre las series analizadas y la línea punteada denota a la estación Yecora con 2 eventos extremos no atribuibles al clima

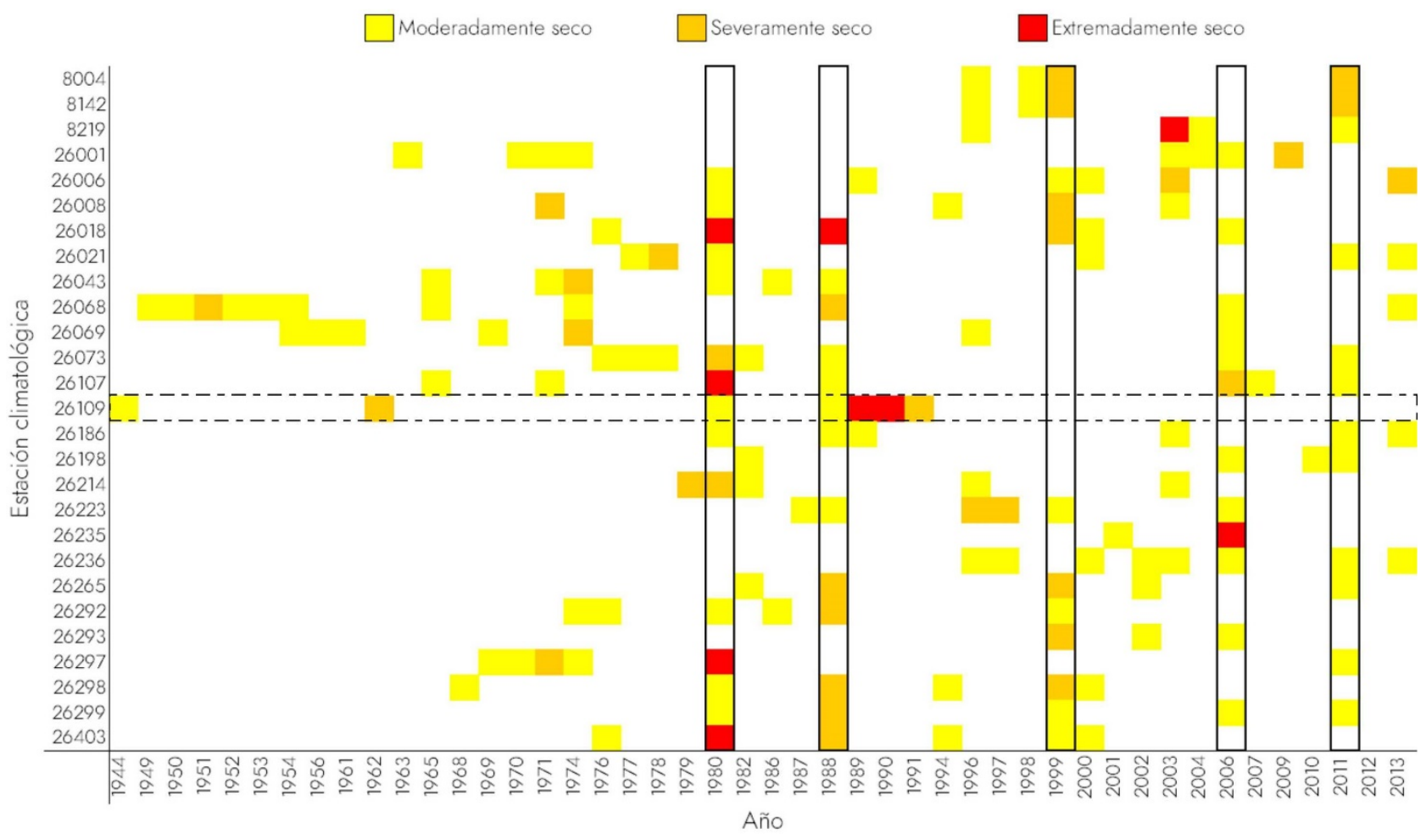

Fuente: elaboración propia a partir del SPI realizadas a las series de datos

Lo anterior indica que los valores obtenidos del SPI son atribuidos a condiciones de instrumentación en la toma de los datos, más que a una condición relacionada con el clima, por 
lo tanto, dicho evento de sequía se consideró inexistente en esta estación para los años 1989 y 1990. La funcionalidad de las estaciones dentro de una cuenca, representan una gran importancia al momento de analizar la sincronía de eventos extremos, con el objetivo de corroborar o validar la información climática, con lo cual se logra un mejor y más apropiado conocimiento de la distribución de la lluvia.

El quinto y sexto año extremos identificados fueron, 2003 en la estación Peñitas (8219) y 2006 en la estación Colonias Yaquis (26235) con un valor extremo de SPI de -2 y -2.4, respectivamente. Para estos dos años las demás estaciones sincronizan con sequías moderadas y otras con sequías severas, por lo cual se infiere que dichos valores obedecen a una variabilidad natural del clima en particular.

Respecto a las sequías severas, se identificó un total de 17 eventos. La mayor parte se distribuye de forma aislada en la cuenca, registrándose un evento o máximo dos en la mayoría de las estaciones. Destacan por su sincronía los años 1988 y 1999, el primero se registró en seis estaciones (26068, 26265, 26292, 26298, 26299 y 26403) las cuales se distribuyen principalmente en la parte baja de la cuenca; y el segundo en siete estaciones (8004, 8142, $26008,26018,26265,26293$ y 26298) la cual tuvo una mayor cobertura espacial, ya que se distribuyen en todo el gradiente de la cuenca, incluso en el área de influencia adicional.

En relación a las sequías moderadas, el primer evento de este tipo se registró en el año de 1944 en la estación Yecora (26109); la segunda ocurre en el año de 1949 en la estación Presa Álvaro Obregón (26068), sequía que se prolonga hasta el año de 1954, un periodo considerado de carencia de precipitación en la parte baja de la cuenca, dado que los registros de esta estación únicamente sincronizan con la estación Presa La Angostura (26069), es difícil analizar el área de impacto de este acontecimiento. La ocurrencia de sequías moderadas y severas, se presentan de forma aislada en la cuenca, registrándose de tres a cinco eventos en la mayoría de las estaciones.

Estudios en México han analizado la variabilidad de la precipitación y caracterizado las sequías utilizando el SPI. Nuñez et al. (2007) analizaron los eventos de sequía para las regiones climáticas más representativas del estado de Chihuahua para el periodo 1970-2004; en la escala de tiempo de 12 meses se identificaron 13 eventos de sequía; los de mayor duración e intensidad ocurrieron a finales de la década de 1990, destacando los ocurridos de diciembre de 1998 a enero del 2001, y de junio de 2002 a septiembre de 2004; ambos manifestaron intensidades entre moderada y extrema. En el Pacífico norte de México para el período 1961- 
2010, se estimó el SPI a escalas de 12 meses, encontrando que los principales episodios de sequía se registraron en la década de 1980 y 2000, en el año de 1982 se registró el episodio de mayor intensidad, aunque el episodio de 2003 presentó una duración y magnitud superior (Serrano et al., 2016). Castillo et al. (2017) calcularon el SPI para la cuenca del río Fuerte al noroeste de México para el período de 1961-2012, el SPI de 12 meses mostró cinco periodos de sequía moderada y una con sequía severa; siendo la más intensa en agosto del año 2000. En un estudio reciente, Esquivel et al. (2019) caracterizaron las sequías en la parte alta de la cuenca del Rio Nazas, Durango, e identificaron que los eventos de sequías más severas y de mayor magnitud se registraron en las décadas de 1950, 2000 y 2010; y sequías moderadas en las décadas de 1970 y 1980.

Estos resultados sincronizan con las sequías identificadas para la CRY, donde las sequías más severas se registraron principalmente en las décadas de 1980 y 2000. Así mismo, cada uno de los años de sequías severas identificadas en este estudio, coinciden con años extremos del Índice de Severidad de Sequía de Palmer (PDSI, por sus siglas en inglés) para el estado de Sonora (1942, 1943-1944, 1946-50, la década de 1950, 1973-74, 1980-81, 1983 y 198788) (Liverman, 1999) y las condiciones de sequía observadas en los mapas de sequía generados con el Atlas de Sequías para México (MXDA, por sus siglas en inglés) (Stahle et al., 2016) (Figura 7). 
Figura 7. Mapas de sequía generados con el Atlas de Sequía para México (MXDA) los cuales indican las condiciones de aridez en los años en los cuales hubo mayor sincronía de eventos entre series de precipitación para la CRY (línea discontinua).

Los valores -6 (color rojo) y 6 (color azul) en la escala PDSI indican condiciones de sequía extrema y humedad extrema

PDSI ANUAL
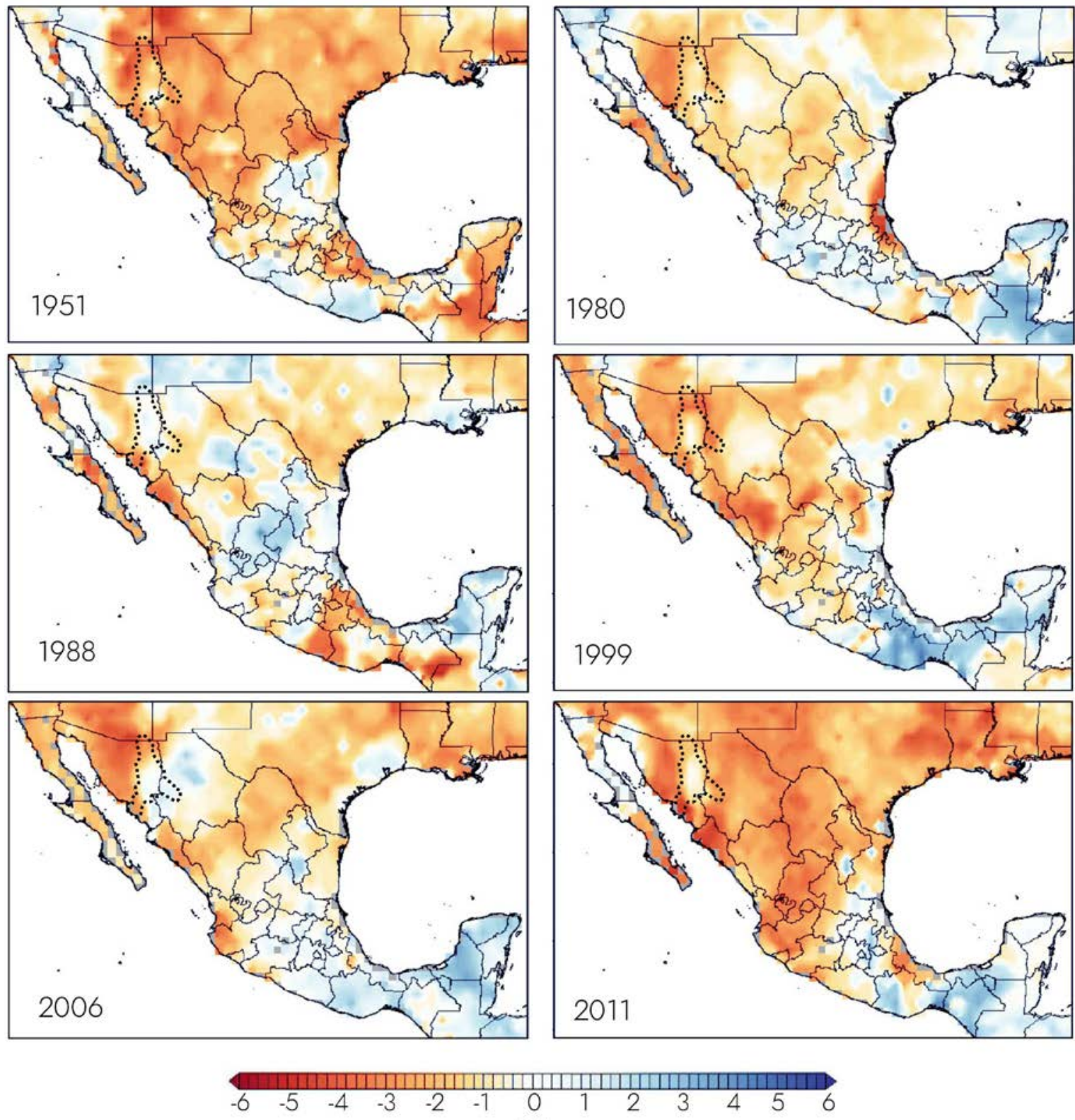

Fuente: elaboración propia a partir del atlas de sequía para México (Stahle et al., 2016)

\subsection{Variabilidad de los valores del SPI}

Se determinó una correlación significativa $(p<0.01)$ entre los valores de SPI de las 27 series de precipitación para la CRY (Figura 8). Un ACP determinó que de las 27 series de SPI, 24 registran la misma variabilidad común. El ACP generó dos componentes principales que 
explican el $60 \%$ de la varianza. El CP1 agrupo 24 series de SPI, las cuales explican el 49.6\% de la varianza (Figura 9).

Figura 8. Análisis de correlación de Pearson entre las series de SPI, los valores remarcados en color rojo indican una alta correlación significativa entre series $(p<0.01)$

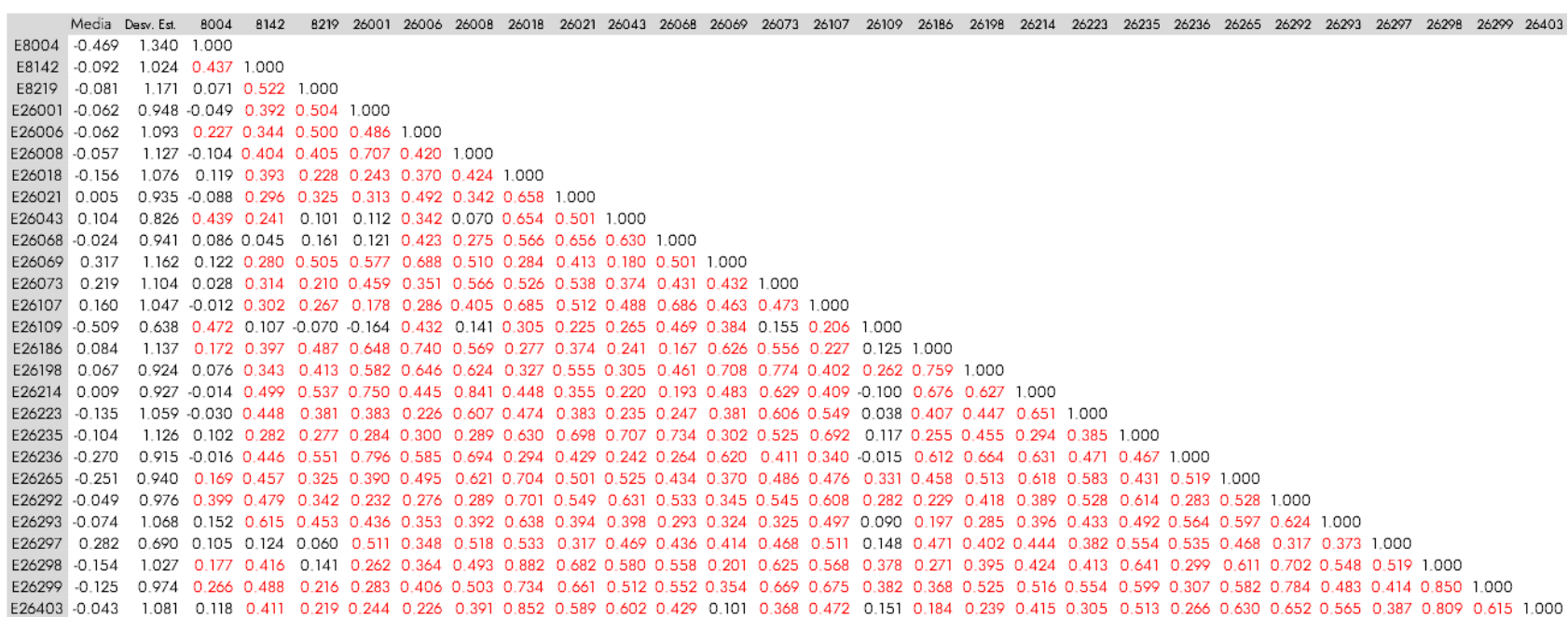

Fuente: elaboración propia a partir de los valores de SPI

Figura 9. Varianza explicada y valores propios de los dos principales componentes del SPI para la CRY. Valores propios para el PC1 (12.10) y CP2 (3.50)

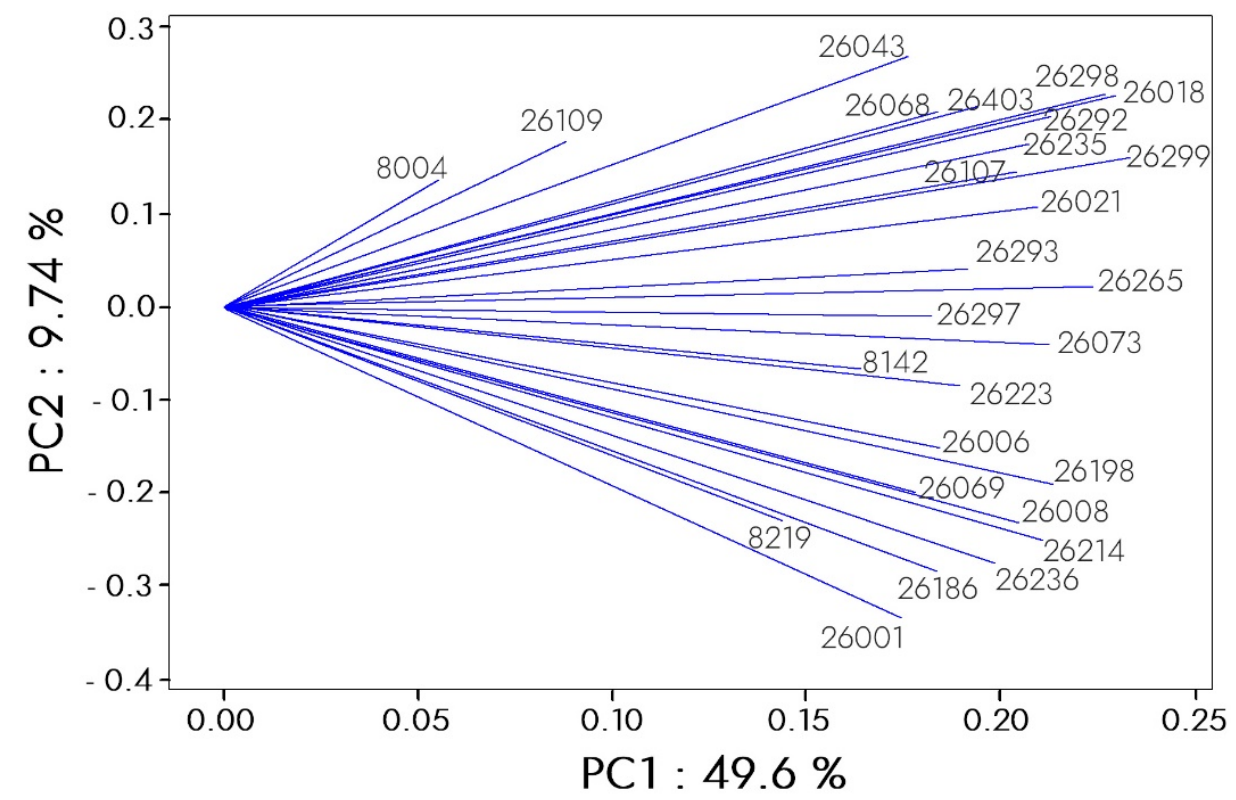

Fuente: elaboración propia a partir del SPI realizadas a las series de datos (SMN, 2019)

Se generó una serie de SPI regional (1937-2016) para la CRY con base en las 24 estaciones registrada en el CP1. EL SPI regional muestra los principales eventos secos y húmedos que se 
han presentado en las últimas décadas, sobresaliendo los eventos ocurridos en los años 1980, 1988, 2006 y 2011 (Figura 10). Así mismo, otros eventos importantes de sequía se observan para las décadas de 1940, 1950, 1970, finales de 1980 y 1998. Cada uno de estos años de sequía han sido reportadas en estudios dendroclimáticos desarrollados tanto en el norte como en el centro del país (Cerano et al., 2012; Cerano et al., 2014; Villanueva et al., 2015; Chávez et al., 2017). La sequía más reciente registrada en el año 2011, es considerada como la peor sequía en las últimas siete décadas para los estados del norte y centro del país, incidiendo en 60\% del territorio mexicano (Domínguez, 2016).

Figura 10. Índice Estandarizado de Precipitación regional (1937-2016) para la CRY

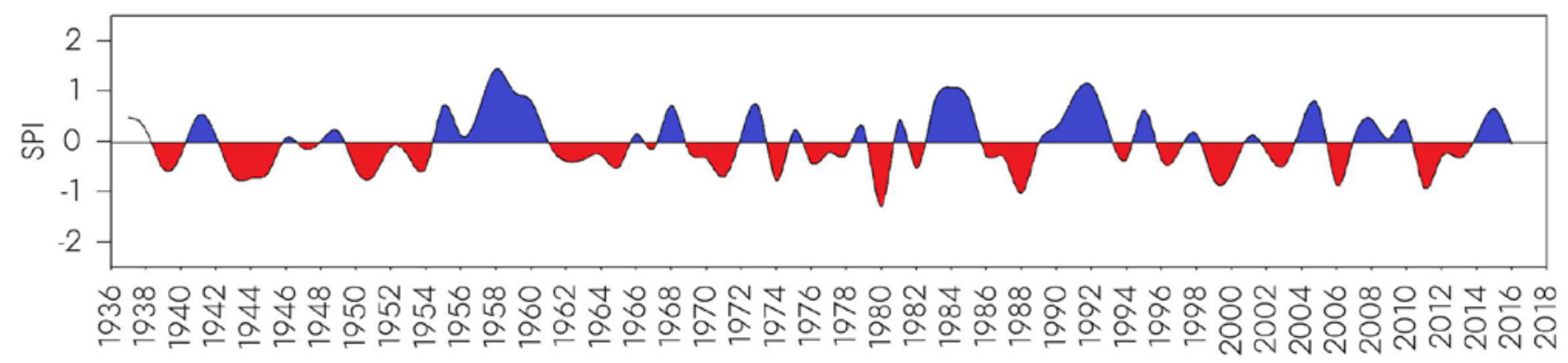

Fuente: elaboración propia a partir del SPI

\subsection{Influencia del ENSO en la variabilidad del SPI}

Un análisis espectral del SPI anual y los índices del Niño 3.4, determinó que el espectro de ondeleta para el SPI muestra áreas significativas $(p<0.05)$ que corresponden a uno y dos años para los períodos 1971-1974 y 1979-1982, de seis a 10 años para el periodo 1981-1992 y de nueve a 11 años para 1954-1960 (Figura 11a). El espectro del Niño 3.4 registra una frecuencia irregular de uno a dos años para el periodo de 1964-1966, de uno a cinco años para 19691974 y de cuatro a siete años para el periodo de 1985-2002 (Figura 11b). Estos resultados son difícil de asociar por separado, aunque las áreas significativas señaladas indican periodicidades comunes que representan períodos secos o húmedos y de presencia o ausencia de ENSO.

La asociación entre ambas variables y las situaciones implicadas pueden identificarse mediante el análisis de coherencia de ondeletas (Figura 11c). Este análisis entre el SPI y Niño 3.4, indica una relación en fase (positiva) significativa $(p<0.05)$ durante el período de 1937 a 1959 a frecuencias de uno a seis años, de 1937 a 1950 en frecuencias de 10 a 11 años, de 1972 a 1974 a frecuencias de uno a dos años y de 1997 a 2005 a frecuencias de 11 a 15 años (Figura 11c). 
Figura 11. Análisis de coherencia de ondeletas entre SPI y ENSO. Las manchas más intensas (rojo) delimitadas por una línea continua indican la presencia de una relación significativa $(p<0.05)$ entre ambas variables. Las flechas horizontales hacia la derecha presentan una relación positiva e indican que ambos fenómenos están en fase, mientras que las flechas en dirección izquierda, denotan una relación negativa o antifase, mientras que las flechas con orientación vertical indican un desfase entre variables

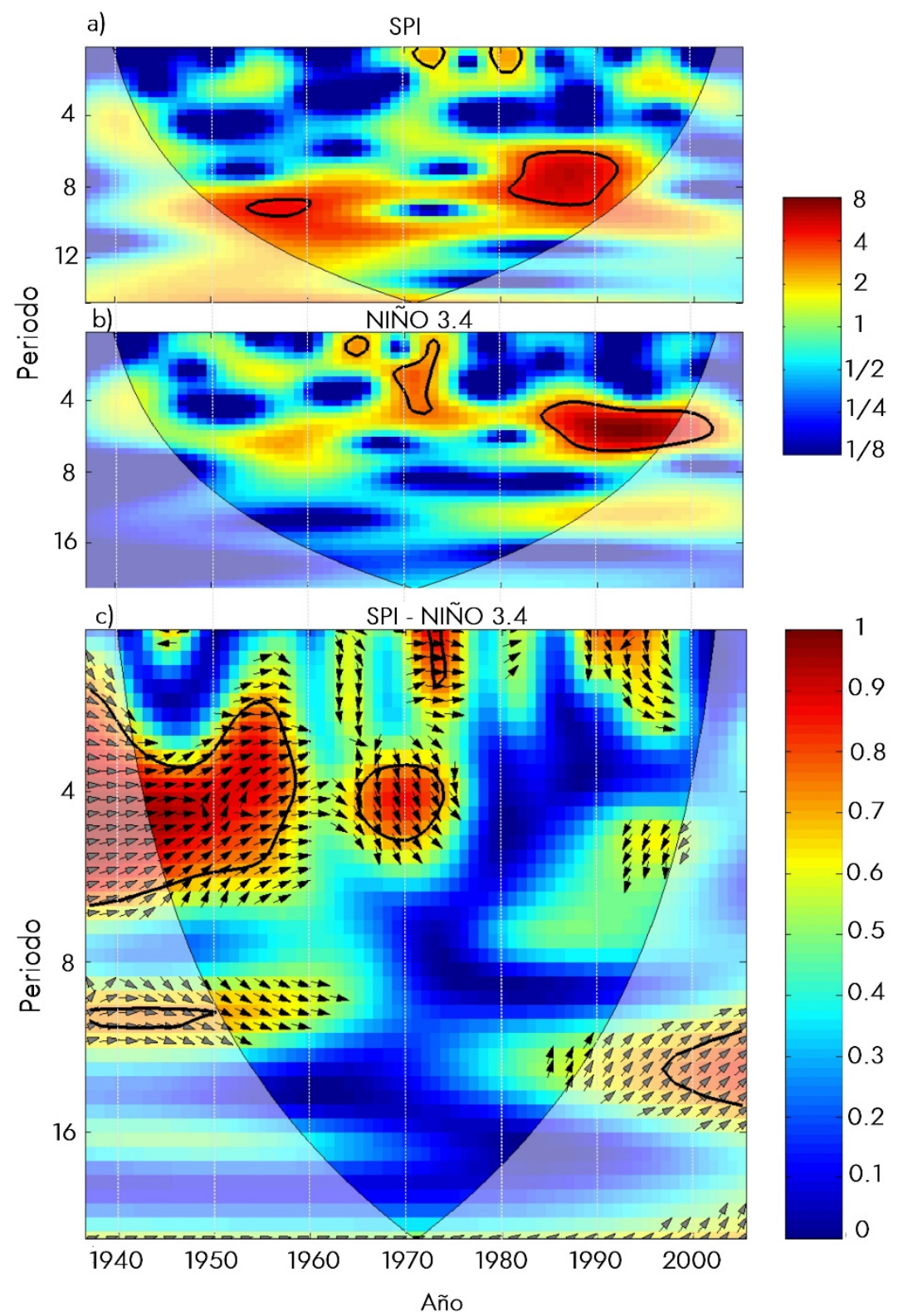

Fuente: elaboración propia a partir del SPI y de la base de datos de ENSO derivado de reconstrucciones climáticas (Li et al., 2013)

El SPI en la cuenca del Río Yaqui es modulado por ENSO, tanto en su fase fría (La Niña) al provocar sequías, como en su fase cálida (El Niño) al propiciar incrementos en la pluviometría de la región. La fase fría de ENSO se relacionó directamente con disminución de la 
precipitación en los períodos 1938-1940, 1942-1946, 1950-1954, 1999-2000 y 20022004. De igual manera, la fase cálida de ENSO ha propiciado incrementos importantes en la precipitación en los años 1940-1942, 1955-1959, 1972-1974, 1997 y 2004-2005.

El fenómeno climático ENSO, es un mecanismo de forzamiento climático que modifica los patrones normales del clima a escala global (Seager et al., 2009). Para el caso de México, este fenómeno tiene una gran influencia en la variabilidad de la precipitación (Magaña et al., 2003). Esquivel et al. (2019) realizaron un análisis espectral entre el SPI y el índice de El Niño 3.4 en la parte alta de la cuenca del Río Nazas, encontrando una relación en fase para los periodos secos y lluviosos registrados en 1920-1930, 1950 y de 1980 a 2005, los cuales han sido influenciados de manera significativa $(p<0.05)$ por la ocurrencia de ENSO en periodos de 2 a 7 años, 2 a 3 años y de 10 a 14 años, respectivamente. Las frecuencias reportadas son similares a lo encontrado en nuestro estudio y se observa una sincronía de los eventos de sequía para las décadas de 1950 y 2000.

Nicholas and Battisti (2008) para la cuenca del Yaqui relacionaron la precipitación anual y estacional con una variedad de índices (Global SST, Regional SST, Gulf of CA SST, Niño-1+2, Niño-3, Niño-3.4, Niño-4, PDO y PNA) detectando una fuerte correlación (0.53) con el índice Niño 3.4 de manera estacional en invierno. En otros estudios, Mosiño y García (1974) mencionan que ENSO se manifiesta en Baja California Norte y Sonora con aumentos en las Iluvias invernales; Douglas y Englehart (1997) lo relacionan con las Iluvias de verano en México.

Cleaveland, Stahle, Therrell, Villanueva y Burns (2003) han documentado la relación entre ENSO y la lluvia para Durango, México, ubicado al sureste de la cuenca del rio Yaqui; Cerano et al. (2011) reconstruyeron para ese mismo estado, la variabilidad de la precipitación en la parte alta de la cuenca del río Nazas, documentando una influencia significativa del ENSO en la modulación de la variabilidad de la precipitación. Este mismo predominio fue encontrado para la precipitación estacional en la región hidrológica Presidio-San Pedro en la vertiente oeste de la SMO en el estado de Durango (Díaz et al., 2016). Otros estudios a nivel global han analizado la influencia de ENSO en la precipitación mediante el uso del SPI; Lovino et al (2014) relacionó los valores de SPI obtenidos para la región noreste de Argentina encontrando sincronía con los eventos La Niña; Sarricolea y Meseguer (2015) efectuaron la caracterización de las sequías en Chile central, los resultados fueron significativos $(p<0.05)$ mostrando una relación directa entre la presencia de El Niño con lluvias abundantes y sequías con La Niña. 


\section{Conclusiones}

La sequía es un riesgo incidente de la naturaleza que puede provocar severos impactos negativos en los diversos sectores productivos y económicos de una región o país, siendo capaz de alterar dramáticamente el desarrollo social y las actividades humanas, así como el medio ambiente. En el caso de México, los impactos de las sequías se han manifestado desde la antigüedad hasta nuestros días y han afectado a los distintos sectores socioeconómicos, pero sobre todo al sector agropecuario y a la población rural que vive en las zonas áridas y semiáridas del país (Gaucin \& Velasco, 2013). Para el caso de la CRY, que fluye desde su origen en la provincia hidrogeológica de la Sierra Madre Occidental hasta el golfo de California y que constituye uno de los mayores sistemas de ríos del noroeste de México, las sequías son uno de los fenómenos que afectan negativamente sus escurrimientos. El $82 \%$ de los escurrimientos se producen en la parte alta de esta cuenca, en ecosistemas con vegetación de bosque mixto, los cuales se conducen a través de afluentes para alimentar y suministrar el agua a una población aproximada de 600 mil personas, la cual habita en las planicies en la parte baja de la cuenca. Ante la relevancia de la cuenca y aunado a la falta de estudios que analicen la variabilidad de la lluvia, intensidad de las sequías, frecuencia y sobre todo, que se logre entender como un fenómeno climático puede propiciar dicha variabilidad, cada nueva investigación que se desarrolle en la CRY y brinde información que permita tener conocimiento y entendimiento de la variabilidad de la lluvia y su relación con fenómenos de circulación general, es un avance de gran relevancia que viene a brindar bases técnicas importantes para fundamentar el desarrollo de programas de manejo, y garantizar la conservación de los servicios ambientales que proporciona esta importante cuenca en la región norte de México.

El presente estudio logró generar una serie de SPI para el periodo 1937-2016 para la CRY a una escala temporal de 12 meses, esta serie permitió analizar la variabilidad temporal e intensidad de las sequías. Se determinó que los eventos de sequías más severas y de mayor cobertura espacial que se han registrado en la CRY se presentaron en los años de 1980, 1988, 1999, 2006 y 2011. Cada una de estas sequías severas identificadas en el presente estudio sincroniza con eventos extremos de sequías observados en otras regiones y cuencas del país, lo que denota su amplitud geográfica y severidad. Así mismo, que son eventos modulados por fenómenos climáticos de circulación general que afectan a gran escala.

Se determinó una relación significativa entre la variabilidad del SPI y el ENSO, los períodos secos y lluviosos más importantes registrados en la CRY han sido influenciados de manera 
significativa por la ocurrencia de ENSO, índices de SPI negativos extremos están relacionados con la fase de La Niña y los índices de SPI con valores positivos más altos se relacionan con la fase de El Niño. Gracias a los avances tecnológicos que se han desarrollado para lograr medir la variabilidad de este fenómeno climático de gran importancia a nivel global, hoy en día se puede conocer con antelación su comportamiento o fase en la cual se desarrollará (Niño o Niña), por lo tanto, si la CRY es una zona donde este fenómeno afecta la variabilidad de la precipitación, algunas veces positivamente y otras de manera negativa, tal como lo demuestran nuestros resultados (una relación en fase significativa entre la variabilidad de este fenómeno climático y el SPI), esto permitirá inferir el comportamiento de la variabilidad climática en la CRY en años futuros y con antelación tomar las medidas adecuadas para disminuir los efectos negativos en sus diferentes ecosistemas y los servicios ambientales que brinda.

Dada la extensión territorial y el gradiente altitudinal de la CRY, se presentan en la región de estudio climas secos, semicálidos y templados, con su consecuente variación en biodiversidad florística y faunística. Sin embargo, esta gran biodiversidad, se ve fuertemente a menazada ante las severas sequías, las cuales propician condiciones de estrés en la vegetación, condición ambiental ideal para que se desarrollen brotes importantes de plagas y enfermedades forestales, lo cual año con año causa la muerte de árboles en cientos de hectareas. Así mismo, las condiciones de sequía dan paso para que se desarrollen incendios severos que ponen en riesgo la biodiversidad de la zona. Se ha determinado que, el fenómeno climático ENSO en el último siglo está relacionado de manera significativa con los niveles más bajos y altos de reservorios de agua en las presas (Cerano et al., 2011), con la propagación de brotes de plagas (Cervantes et al., 2019) y con el desarrollo de los incendios más severos (Cerano et al., 2019).

Toda esta información da una idea de la magnitud de las fases de ENSO en la modulación de la variabilidad de la precipitación, sus efectos en los ecosistemas y los servicios ambientales. Estas condiciones y efectos se pueden repetir y magnificar ante futuros escenarios de las fases de ENSO. Los resultados de los estudios indicados anteriormente, aunado a los resultados de esta investigación, permiten entender la magnitud de los efectos de dicho fenómeno, siendo vital considerar las medidas pertinentes de manejo en cada uno de los diferentes ecosistemas de la cuenca ante futuros pronósticos de las fases en que se desarrollará el ENSO.

La información generada en esta investigación es de alta relevancia para los tomadores de decisiones en la administración del recurso hídrico de los embalses establecidos en el gradiente de la cuenca, ya que son los responsables de manejar la distribución del agua en los distritos de 
riego y proponer el patrón óptimo de cultivos dada cierta disponibilidad de agua. En el caso del personal administrador encargado de las áreas naturales protegidas, ya que en términos ambientales sufren de pérdida de flora y faunas silvestres, erosión y pérdida de suelos, degradación de la calidad del agua, así como la pérdida de servicios ecosistémicos; respecto a los manejadores de las áreas forestales, dada la vulnerabilidad de la región a incendios forestales y potencial brote de plagas, entre otros. Todos deben considerar los pronósticos de desarrolló de ENSO, con el objetivo de disminuir el riesgo asociado a los eventos de sequía, lo cual puede contribuir a garantizar una mejor planeación y por lo tanto lograr mejores resultados de los programas de manejo de los recursos de la cuenca ante las diferentes fases de ENSO y con la firme convicción de disminuir los estragos ecológicos y por consiguiente los efectos sociales y económicos.

Agradecimientos: Esta investigación fue posible gracias al financiamiento a través del proyecto "Procesos de mitigación del impacto por cambio climático en la agricultura del sur de Sonora", con clave 1450034669. Asimismo, agradecemos a los revisores anónimos por sus comentarios y observaciones que contribuyeron a mejorar el manuscrito.

Declaración responsable: Las/os autoras/es declaran que no existe ningún conflicto de interés con relación a la publicación de este artículo. La participación de los autores se ha distribuido de la siguiente manera: Julián Cerano Paredes y Gerardo Esquivel Arriaga: desarrollaron el planteamiento del estudio, la metodología, análisis estadístico, resultados y conclusiones. Josué Raymundo Estrada Arellano: trabajo directamente en la introducción y revisión de literatura. Ignacio Sánchez Cohen: trabajo en la revisión de literatura y conclusiones. 


\section{Bibliografía}

Alexandersson, H. (1986). A homogeneity test applied to precipitation data. Journal of Climate, 6, 661-675.

Alexandersson, H., \& Moeberg, A. (1997). Homogeneization of Swedish temperature data. Part I: Homogeneity test for linear trends. International Journal of Climatology, 17, 25-34.

Andreoli, R. V., \& Kayano, M. T. (2004) Multi-scale variability of the sea surface temperature in the Tropical Atlantic. Journal of Geophysical Research Oceans, 109, C05009, https://doi.org/10.1029/2003/C002220

Barakat, F., \& Handoufe, A. (1998). Approche Agroclimatique de la Sécheresse Agricole au Maroc. Sécheresse, 9(3), 201-208.

Bootsma, A., Boisvert, J., \& Baier, R. (1996). La Sécheresse et L'agriculture Canadienne: une Revue des Moyens D'action. Sécheresse, 7(4), 277-285.

Brunet, I. M. (2010). Datos e indicadores para detectar y atribuir eventos al cambio climático: los registros históricos del clima y su problemática. Realidad, datos y espacio. Revista internacional de estadística y geografía, 1(1), 16-25. Retrieved from https://rde.inegi.org.mx/rde_01/doctos/rde_01_art3.pdf

Buishand, T. A. (1982). Some methods for testing the homogeneity of rainfall records. Journal of Hydrology, 58, 11-27.

Canchala, T., Loaiza, C. W., Francés, F., Carvajal, E., Y., Valéria, A., R., Toshie, K. M., Alfonso, M. W., Caicedo, B. E., \& Ferreira de Souza, R. A. (2020). Streamflow Variability in Colombian Pacific Basins and Their Teleconnections with Climate Indices. Water, 12(526); https://doi.org/10.3390/w12020526

Castillo, C. M., Ibañez, C. L. A., Valdés J. B., Arteaga R. R., \& Vázquez, P. M. A. (2017). Análisis de sequías meteorológicas en la cuenca del Río Fuerte, México. Tecnología y Ciencias del Agua, 8(1), 35-52. Retrieved from http://www.scielo.org.mx/pdf/tca/v8n1/2007-2422-tca8-01-00035.pdf

Cerano P. J., Villanueva D. J., Valdez C. R. D., Arreola A. J. G., \& Constante G. V. (2011). El niño oscilación del sur y sus efectos en la precipitación en la parte alta de la cuenca del río Nazas. Revista Chapingo Serie Ciencias Forestales y del Ambiente, 18, 207215. https://doi.org/10.5154/r.rchscfa.2010.09.076 
Cerano, P. J., Villanueva, D. J., Valdez, C. R. D., Constante, G. V., González, B. J. L., \& Estrada, A. J. (2012). Precipitación reconstruida para la parte alta de la cuenca del río Nazas, Durango. Revista Mexicana de Ciencias Forestales, 3(10), 7-23. Retrieved from http://www.scielo.org.mx/pdf/remcf/v3n10/v3n10a2.pdf

Cerano, J. P., Díaz, J. V., Martínez, R. C., Selem, L. V., Caciano, R. T., \& De la Cruz, V. G. (2014). Reconstrucción de precipitación invierno-primavera para el Parque Nacional Pico de Tancítaro, Michoacán. Investigaciones

Geográficas,

(83),

\section{4. https://doi.org/10.14350/rig.35190}

Cerano-Paredes, J., Villanueva-Díaz, J., Vázquez-Selem, L., Cervantes-Martínez, R., MagañaRueda, V. O., Constante-García, V., Esquivel-Arriaga, G., \& Valdez-Cepeda, R. D. (2019). Climatic influence on fire regime (1700 to 2008) in the Nazas watershed, Durango, Mexico. Fire Ecology, 15(1), 9.

Cervantes-Martinez, R., Cerano-Paredes, J., Sánchez-Martínez, G., Villanueva-Diaz, J., EsquivelArriaga, G., Cambrón-Sandoval, V. H., Méndez-González, J., \& Castruita-Esparza, L. U. (2019). Historical bark beetle outbreaks in Mexico, Guatemala and Honduras (1895-2015) and their relationship with droughts. Revista Chapingo Serie Ciencias Forestales, 25(2). Retrieved from: https://chapingo-cori.mx/revistas/articulos/doc/r.rchscfa.2019.01.006.pdf

Chávez G. M. P., Cerano P. J., Nájera L. J. A., Pereda B. V., Esquivel A. G., Cervantes M. R., Cambrón S. V. H., Cruz C. F., \& Corral R. S. (2017). Reconstrucción de la precipitación invierno-primavera con base en anillos de crecimiento de árboles para la región de San Dimas, Durango, México. BOSQUE, 38(2), 387-399, https://doi.org/10.4067/S0717$\underline{92002017000200016}$

Cleaveland, M. K., Stahle D. W., Therrell, M. D., Villanueva, D. J., \& Burns, B. T. 2003. Treering reconstructed winter precipitation and tropical teleconnections in Durango, Mexico. Climatic Change, 59, 369-388. Retrieved from https://www.researchgate.net/publication/285600666_Tree-

Ring_Reconstructed_Winter_Precipitation_and_Tropical_Teleconnections_in_Durango_Mexico Comisión Estatal del Agua (CEA) (2008). Estadísticas del agua en Sonora. CEA: México, D. F. CONAGUA (Comisión Nacional del Agua) (2017). Estadísticas agrícolas de los distritos de riego. Año agrícola 2015-2016. Secretaría de Medio Ambiente y Recursos Naturales - 
CONAGUA Subdirección General de Planeación, Ciudad de México, México. Retrieved from https://files.conagua.gob.mx/conagua/publicaciones/Publicaciones/EA_2015-2016.pdf

CONAGUA (Comisión Nacional del Agua) (2018). Estadísticas del Agua en México, edición 2018. Secretaría de Medio Ambiente y Recursos Naturales - CONAGUA Subdirección General de Planeación, Ciudad de México, México. Retrieved from http://sina.conagua.gob.mx/publicaciones/EAM_2018.pdf

Cotler, H. (2010). Las cuencas hidrográficas de México. Diagnóstico y Priorización. SEMARNAT, Fundación Gonzalo Río Arronte, México, D. F.

Davydova, B. V. (2010). Variabilidad climática y sus efectos sobre las cuencas de México. In H. Cotler, Las cuencas hidrográficas de México. Diagnóstico y Priorización (pp. X28-31). SEMARNAT, Fundación Gonzalo Río Arronte, México, D. F.

Díaz, R. B., Villanueva, D. J., \& Cerano, P. J. (2016). Reconstrucción de la precipitación estacional con anillos de crecimiento para la región hidrológica Presidio-San Pedro. Madera y Bosques, 22(1), 111-123 Retrieved from http://www.scielo.org.mx/pdf/mb/v22n1/1405-0471mb-22-01-00111.pdf

DOF, Diario Oficial de la Federación (2001). Acuerdo secretarial de re-categorización de la reserva Forestal Nacional y Zona de Refugio de la Fauna Silvestre Tutuaca, como área de Protección de Flora y Fauna Tutuaca. SEMARNAT-Diario Oficial de la Federación. Retrieved from http://dof.gob.mx/nota_detalle.php?codigo=757569\&fecha=27/12/2001

DOF, Diario Oficial de la Federación (2003). Aclaración de re categorización del área de protección de recursos naturales, como área de Protección de Flora y Fauna Campo Verde. SEMARNAT-Diario Oficial de la Fedrieved from http://dof.gob.mx/nota_detalle.php?codigo=706407\&fecha=29/01/2003

DOF, Diario Oficial de la Federación (2017). Acuerdo para dotar con la categoría de Área de Protección de Flora y Fauna a la Reserva Forestal Nacional y Refugio de Fauna Silvestre Bavispe. SEMARNAT-Diario Oficial de la Fetrieved from https: / / www.dof.gob.mx/nota_detalle.php?codigo $=5483731 \& f e c h a=22 / 05 / 2017$

Domínguez, J. (2016). Revisión histórica de las sequías en México: de la explicación divina a la incorporación de la ciencia. Tecnología y ciencias del agua, 7(5), 77-93 Retrieved from http://www.scielo.org.mx/pdf/tca/v7n5/2007-2422-tca-7-05-00077.pdf 
Douglas, A. V., \& Englehart, P. J. (1997). Inter-monthly variability of the Mexican summer monsoon. Twenty Second Annual Climate Diagnostics and Prediction Workshop. NOAA. USA.

Entekhabi, D., Rodríguez-lturbide, I., \& Brass, R. L. (1992). Variability in Large-scale Water Balance with Land Surface-Atmosphere Interaction. Journal of Climate, 5, 798813. https://doi.org/10.1175/1520-0442(1992)005<0798:VILSWB>2.0.CO;2

Escobar, P. J. J., González, B. J. L., Sánchez, C. I., Muñoz, V. A., \& Villa, C. M. (2012). Análisis de la lluvia y su relación con fenómenos del cambio climático; estudio de caso en estación meteorológica Sardinas (San Bernardo, Durango). Revista Chapingo Serie Zonas Áridas, 11(2), 75-84. Retrieved from https://www.redalyc.org/articulo.oa?id=455545059003

Esquivel, A. G., Cerano, P. J., Sánchez, C. I., Velásquez, V. M. A., Flores, L. F., \& Bueno, H. P. (2019). Análisis temporal de sequías (1922-2016) en la cuenca alta del río Nazas usando el SPI y su relación con ENSO. Tecnología y Ciencias del Agua, 10(3), 126153. https://doi.org/10.24850/j-tyca-2019-05-05

García A. de M. E. (2003). Distribución de la precipitación en la República Mexicana. Investigaciones Geográficas. Boletín del Instituto de Geografía UNAM, 50, 67-76 Retrieved from http://www.scielo.org.mx/pdf/igeo/n50/n50a9.pdf

GEC (Gobierno del estado de Chihuahua) (2019). Secretaria de desarrollo urbano y ecología. http://www.chihuahua.gob.mx/sedue/areas_protegidas_chih

Grinsted, A., Moore, J. C., \& Jevrejeva, S. (2004). Application of the cross wavelet transform and wavelet coherence to geophysical time series. Nonlinear Proc. Geophys. 11, 561566 https://doi.org/10.5194/npg-11-561-2004

Guajardo, P. R. A., Granados, R. G. R., Sánchez, C. I., Díaz, P. G., \& Barbosa, M. F. (2017). Validación espacial de datos climatológicos y pruebas de homogeneidad: caso Veracruz, México. Tecnología y Ciencias del Agua, 8(5), 157-177. https://doi.org/10.24850/j-tyca-2017$\underline{05-11}$

Hainie, A. N \& Sayang M. D. S. (2013). Homogeneity Test on Daily Rainfall Series for Malaysia. Matematika, 29(1c), $141-150$ Retrieved

from https://matematika.utm.my/index.php/matematika/article/download/586/579

Helena, B., Pardo, R., Vega, M., Barrado, E., Fernandez, J.M., \& Fernandez, L. (2000). Temporal evolution of groundwater composition in an alluvial aquifer (Pisuerga River, Spain) by 
principal component analysis. Water Research, 34, 807-816 https://doi.org/10.1016/S00431354(99)00225-0

Heim, R. R. Jr. (2002). A review of twentieth-century drought indices used in the United States. Bulletin of the American Meteorological Society, 83, 1149-1165. Retrieved from https://pdfs. semanticscholar.org/8790/277954cd5985b244a7522333263789ff242c.pd $\underline{f}$

INEGI (2000). Síntesis de Información Geográfica del Estado de Sonora: Aguascalientes, Ags. Instituto Nacional de Estadística, Geografía e Informática, Secretaría de Programación y Presupuesto. Retrieved from

http://internet.contenidos.inegi.org. mx/contenidos/Productos/prod_serv/contenidos/espanol /bvinegi/productos/historicos/2104/702825223328/702825223328_1.pdf

Jolliffe, I. T., \& Cadima, J. (2016). Principal component analysis: A review and recent developments. Philosophical Transactions of the Royal Society A, 374, 20150202 https://doi.org/10.1098/rsta.2015.0202

Kaplan, A., Cane, M., Kushnir, Y., Clement, A., Blumenthal, M., \& Rajagopalan, B. (1998). Analyses of global sea surface temperature 1856-1991. Journal of Geophysical Research, 103, C9, 18, 567-18,590, https://doi.org/10.1029/97JC01736

Li, J., Shang-Ping, X., Cook, E. R., Morales, M. S., Christie, D. A., Johnson, N. C., Chen, F., D'Arrigo, R., Fowler, A. M., Gou, X., \& Fang, K. (2013). El Niño modulations over the past seven centuries. Nature climate change, 3, 822-825. https://doi.org/10.1038/NCLIMATE1936 Liverman, D. M. (1999). Vulnerability and adaptation to drought in Mexico. Natural Resources Journal, 39(1), 99-115 Retrieved from

https://digitalrepository.unm.edu/cgi/viewcontent. cgi? referer=\&httpsredir=1\&article=1658\&co $\underline{\text { ntext=nrj }}$

Lovino, M., García, N. O., \& Baethgenc, B. (2014). Spatiotemporal analysis of extreme precipitation events in the Northeast region of Argentina (NEA). Journal of Hydrology: Regional Studies, 2, 140-158. http://dx.doi.org/10.1016/j.ejrh.2014.09.001

Lutz, A., \& Kurl, K. (2012). Panorama de la cuenca del Rio Yaqui. (Information document). InterAmerican Institute for Global Change Research. Retrieved from http://aquasec.org/wrpg/wp-content/uploads/2012/10/YaquiFactSheet-

SpanishFINAL.pdf 
Magaña, R., Pérez J. L., Conde, C., Gay, C., \& Medina, S. (1997). El fenómeno de El Niño y la Oscilación del Sur (ENOS) y sus impactos en México. Departamento de Meteorología General. Centro de Ciencias de la Atmósfera. México, D. F.: Universidad Nacional Autónoma de México. Magaña, R. V. O., Vásquez, J. L., Pérez, J. L., \& Pérez, J. B. (2003). Impact of El Niño on precipitation in Mexico. Geofísica Internacional, 42, 313-330.

Marcos, V. O. (2001). Sequía: definiciones, tipologías y métodos de cuantificación. $\begin{array}{llll}\text { Investigaciones } \quad \text { geográficas, } & \text { 26, } & \text { Retrieved }\end{array}$ from https://dialnet.unirioja.es/descarga/articulo/111741.pdf

Martínez, A. P. F., Patiño, G. C., \& Tamayo, E. J. E. (2014). Análisis de tendencias climáticas en la cuenca baja del río Yaqui, Sonora, México. Tecnología y Ciencias del Agua, 5(6), 135-149. Retrieved from http://www.scielo.org.mx/pdf/tca/v5n6/v5n6a9.pdf

MathWorks (2002). MATLAB Versión 6.5 (Mathematics software).

McKee, T. B., Doesken, N. J., \& Kleist, J. (1993). The relationship of drought frequency and duration to time scale. In Proceedings of the Eighth Conference on Applied Climatology (pp. 179184). Anaheim, California. American Meteorological Society, Boston. Retrieved from http://www.droughtmanagement.info/literature/AMS_Relationship_Drought_Frequency_D uration_Time_Scales_1993.pdf

Mesbahzadeh, T., Mirakbari, M., Saravi, M. M., Sardoo, F. S., \& Miglietta (2020). Meteorological drought analysis using copula theory and drought indicators under climate change scenarios (RCP). Meteorolical Applications, 27(1), 1 20. https://doi.org/10.1002/met. 1856

Mirakbari, M., Ganji, A., \& Fallah, S. R. (2010). Regional bivariate frequency analysis of meteorological droughts. Journal of Hydrologic Engineering, 15(12), 985 1000. https://doi.org/10.1061/(ASCE)HE.1943-5584.0000271

Modaresi R. A., Ghahraman, B., Khalili, D., Ghahremani, Z., \& Ahmadi, A. S. (2017). Integrated meteorological and hydrological drought model: a management tool for proactive water resources planning of semi-arid regions. Advances in Water Resources, 107, 336353. https://doi.org/10.1016/j.wace.2014.03.007

Morales, G. A., Olcina, C. J., \& Rico, A. A. M. (2000). Diferentes percepciones de las sequías en España: adaptación, catastrofismo e intentos de corrección. Investigaciones Geográficas, 23, 5-46. Retrieved from https://www.redalyc.org/pdf/176/17602301.pdf 
Mosiño, P., \& García, E. (1974). The climate of Mexico. Climates of North America. World Survey of Climatology, 11, 345-404.

Muñoz, A. F., Avissar, R., Zhu, C., \& Lettenmaier, D. P. (2009). Sensitivity of the water resources of Rio Yaqui Basin, Mexico, to agriculture extensification under multiscale climate conditions, Water Resources Research, 45, 1-13. https://doi.org/10.1029/2007WR006783

NDMC (National Drought Mitigation Center) (2019). SPI SL 6.exe Program to Calculate Standardized Precipitation Index. National Drought Mitigation Center. Retrieved from https://drought.unl.edu/droughtmonitoring/SPI/SPIProgram.aspx

Nicholas, R. E., \& Battisti, D. S. (2008). Drought Recurrence and Seasonal Rainfall Prediction in the Rio Yaqui Basin, Mexico. Journal of Applied Meteorology and Climatology, 47, 9911005. https://doi.org/10.1175/2007JAMC1575.1

Núñez, L. D., Muñoz, R. C. A., Reyes, G. V. M., Velasco, V. I., \& Gadsden, E. H. (2007). Caracterización de la sequía a diversas escalas de tiempo en Chihuahua, México. Agrociencia, 41, 253-262 Retrieved from http://www.scielo.org.mx/pdf/agro/v41n3/1405-3195-agro-4103-253.pdf

OMM, Organización Meteorológica Mundial (2012). Índice normalizado de precipitación. Guía del usuario. Ginebra Suiza. Organización Meteorológica Mundial. Retrieved from http://www.droughtmanagement.info/literature/WMO_standardized_precipitation_index_ user_guide_es_2012.pdf

OMM, Organización Meteorológica Mundial (2006). Vigilancia y Alerta Temprana de la Sequía: Conceptos, Progresos y Desafíos Futuros (OMM-No 1006). Retrieved from https://www.droughtmanagement.info/literature/WMO_drought_monitoring_early_warnin g_es_2006.pdf

Ortega, G. D. (2012). Sequía en Nuevo León, Vulnerabilidad, Impactos y Estrategias de Mitigación. Instituto del Agua del Estado de Nuevo León, Mexico. Retrieved from https://www.researchgate.net/publication/260163853_Sequia_en_Nuevo_Leon_Vulnera bilidad_Impactos_y_Estrategias_de_Mitigacion

Paredes, F. J., Barbosa, A. H., \& Guevara, E. (2015). Análisis espacial y temporal de las sequías en el nordeste de Brasil. Agriscientia, 32(1), 1-14. Retrieved from https://revistas.unc.edu.ar/index.php/agris/article/view/16552 
Peterson, T. C., Hoerling, M. P., Stott, P. A., \& Herring, S. (2013). Explaining extreme events of 2012 from a climate perspective. Bulletin of the American Meteorological Society, 94(9), 1-74.

Pettitt, A. N. (1979). A nonparametric approach to the change point detection. Applied Statistics, $28,126-135$.

Ravelo, A. C, Sanz, R. R., \& Douriet, C. J. C. (2014). Detecting, assessing and forecasting droughts in the region of the North Pacific Watershed Organism, Mexico. Agriscientia, 31(1), 114. Retrieved from https://revistas.unc.edu.ar/index.php/agris/article/view/9836

Raziei, T., Martins, D. S., Bordi, I., Santos, J. F., Portela, M. M., Pereira, L. S., \& Sutera, A. (2014). SPI Modes of Drought Spatial and Temporal Variability in Portugal: Comparing Observations, PTO2 and GPCC Gridded Datasets. Water Resources Management, 29(2), 487504. https://doi.org/10.1007/s11269-014-0690-3

Rivera, del R. R., Crespo, P. G., Arteaga, R. R., \& Quevedo, N. A. (2007). Comportamiento espacio temporal de la sequía en el estado de Durango, México. Terra Latinoamericana, 25(4), 383-392. Retrieved from https://www.redalyc.org/pdf/573/57315558006.pdf

Sarricolea, P., \& Meseguer, R. O. (2015). Sequías en Chile central a partir de diferentes índices en el período 1981-2010. Investigaciones Geográficas Chile, 50, 19-32. Retrieved from https://investigacionesgeograficas.uchile.cl/index.php/lG/article/view/41178/42715

SMN (Servicio Meteorológico Nacional) (2019). Información estadística climatológica en formato KMZ. Retrieved from https://smn.conagua.gob.mx/es/climatologia/informacionclimatologica/informacion-estadistica-climatologica

Seager, R., Ting, M., Davis, M., Cane, M., Naik, N., Nakamura, J., Li, C., Cook, E., \& Stahle, D. W. (2009). Mexican drought: an observational modeling and tree ring study of variability and climate change. Atmósfera, 22, 1-31.

Serrano, B. L., Vicente, S. S. M., Flores, M. H., Tijerina C. L., \& Vázquez, S. D. (2016). Variabilidad espacio-temporal de las sequías en la cuenca pacífico norte de México (1961-2010). Cuadernos de Investigación Geográfica, 42(1), 185-204. https://doi.org/10.18172/cig.2857

Sivakumar, M. V. K., Stefanski, R., Bazza, M., Zelaya, S., Wilhite, D., \& Rocha, M. A. (2014). High level meeting on national drought policy: Summary and major outcomes. Weather and Climate Extremes, 3, 126-132. http://dx.doi.org/10.1016/j.wace.2014.03.007 
Stahle, D. W., Burnette, D. J., Villanueva, D. J., Heim Jr, R. R., Fye, F. K., Cerano, P. J., Acuña, S. R., \& Cleaveland, M. K. (2011). Pacific and Atlantic influences in Mesoamerican over the past millennium. Climate Dynamics, 39(6): 1431-1446. https://doi.org/10.1007/s00382-011-1205-z Stahle D. W., Cook, E. R., Burnette, D. J., Villanueva, D. J., Cerano, J., Burns, J. N., Griffin, R. D., Cook, B. I., Acuña, R., Torbenson, M. C. A., \& Sjezner, P. (2016). The Mexican Drought Atlas: Tree-Ring Reconstructions of the Soil Moisture Balance During the Late Pre-Hispanic, Colonial, and Modern Eras. Quaternary Science Reviews, 149, 34-60 Retrieved from http://drought.memphis.edu/MXDA/

Torrence, C., \& Compo, G. P. 1998. A practical guide to wavelet analysis. Bulletin of the American Meteorological Society, 79(1), 61-78 Retrieved from: https://paos.colorado.edu/research/wavelets/bams_79_01_0061.pdf

Vega, G. E. L.; Cirett, G. S., De la Parra, V. M. L., \& Zavala, J. Raúl. (2011). Hidrogeología de Sonora, México. In T. Calmus (Ed.), Panorama de la geología de Sonora, México (pp. 267298). Universidad Nacional Autónoma de México, Instituto de Geología.

Velasco, I. (2006). Modelo para la detección y evaluación de la sequía en la Cuenca del Río Conchos, México, mediante un SIG. In XXII Congreso Latinoamericano de Hidráulica. Ciudad Guayana, Venezuela.

Villanueva, D. J., Cerano, P. J., Fulé, P. Z., Cortés, M. C., Vázquez, S. L., Yocom, L. L., \& RuizCorral, J. A. (2015). Cuatro siglos de variabilidad hidroclimática en el noroeste de Chihuahua, México, reconstruida con anillos de árboles. Investigaciones geográficas, (87), 141 153. https://doi.org/10.14350/rig.44485

Wilhite, D. A., \& M. H. Glantz (1985). Understanding the Drought Phenomenon: the Role of Definitions. Water International, 10(3) 111-120. Retrieved from https://digitalcommons.unl.edu/droughtfacpub/20/

Yacoub, E., \& Tayfur, G. (2017). Evaluation and assessment of meteorological drought by different methods in Trarza region, Mauritania. Water Resources Management, 31, 825845. https://doi.org/10.1007/s11269-016-1510-8

Zhong, L., Hua, L., \& Yan, Z. (2020). Datasets of meteorological drought events and risks for the developing countries in Eurasia. Big Earth Data, 4(2), 191-223. Retrieved from https://www.tandfonline.com/doi/pdf/10.1080/20964471.2019.1710383 
Zucarelli, G. V. (2013). Identificación de eventos hídricos extremos en la cuenca del río Paraná. Tecnología y Ciencias del Agua, 4(5), 181-187. Retrieved from http://www.scielo.org.mx/pdf/tca/v4n5/v4n5a12.pdf 